\title{
Existence Theorems for Abstract Multidimensional Control Problems ${ }^{1}$
}

\author{
LAMBerto Cesari ${ }^{2}$
}

\begin{abstract}
In the present paper, the author discusses an abstract formulation of control problems involving general operators $\mathscr{L}: S \rightarrow V, \mathscr{M}: S \rightarrow Y$ from a Banach space $S$ into space $V$ and $Y$ of vector functions in a fixed domain with components in $L_{p}, p \geqslant 1$. For this general formulation, the author states closure theorems, lower closure theorems, and existence theorems for an optimal solution. It is then shown that the problems of control involving Dieudonné-Rashevski partial differential equations previously considered by the author are particular cases of the present formulation. Finally, it is shown by examples that problems of control involving usual partial differential equations, linear or not, as well as other functional relations, can be framed in the present formulation. The present work concerns problems with distributed controls. Work concerning problems with distributed as well as boundary controls is forthcoming.
\end{abstract}

\section{Introduction}

We present here existence theorems for multidimensional optimal control problems in an abstract setting, which are extensions of theorems proved in a concrete form in previous papers (Refs. 1-4). The present formulation for general Lagrange problems includes also a number of results which have appeared before for free problems only (Refs. 5-11). The present formulation concerns only distributed control problems in the terminology of Lions (Ref. 12). Extensions including boundary control problems will be discussed elsewhere.

${ }^{1}$ Paper received December 19, 1969; in revised form, January 29, 1970. Parts of this paper were read at the International Conference of Optimal Control, Tbilisi, Georgia, USSR, 1969, and at the Conference on Optimal Control, Ann Arbor, Michigan, 1969 (Tenth Annual Meeting of the Society for Natural Philosophy). This research was partially supported by AFOSR Research Project No. 69-1662.

${ }^{2}$ Professor of Mathematics, University of Michigan, Ann Arbor, Michigan. 
We are interested in control problems where the state variable is an element of a Banach space $S$ with norm $\|x\|$, where $\mathscr{L}: S \rightarrow V$ and $\mathscr{U}: S \rightarrow Y$ are two operators, $\mathscr{L}$ possibly unbounded, $V$ and $Y$ functions spaces of elements $y(t)=\left(y^{1}, \ldots, y^{s}\right), v(t)=\left(v^{1}, \ldots, v^{r}\right), t \in G$, and where the controls are also vector functions $u(t)=\left(u^{1}, \ldots, u^{m}\right), t \in G, G$ a bounded open subset of $E_{v}, \nu \geqslant 1$. Thus, we consider control problems monitored by a functional equation of the form

$$
(\mathscr{L} x)(t)=f(t,(\mathscr{U} x)(t), u(t)) \quad \text { a.e. in } G,
$$

with usual constraints

$$
(t,(\mathscr{U} x)(t)) \in A, \quad u(t) \in U(t,(\mathscr{U} x)(t)) \quad \text { a.e. in } G,
$$

and functional

$$
I[x, u]=\int_{G} f_{o}(t,(\mathscr{U} x)(t), u(t)) d t
$$

Details and more general formulations will be indicated below. Whenever $S$ is a space of vector functions on $G$ and $\mathscr{L}$ and $\mathscr{U}$ are differential operators, then (1) reduces to a usual differential system in $G$.

\section{Abstract Functional Equation}

Let $G$ be a given open bounded subset of the $t$-space $E_{\nu}, t=\left(t^{1}, \ldots, t^{v}\right)$, $v \geqslant 1$, let $Y$ be a space of $s$-vector functions $y(t)=\left(y^{1}, \ldots, y^{s}\right), t \in G$, whose components $y^{i}$ are $L_{p_{i}}$-integrable in $G, p_{i} \geqslant 1, i=1, \ldots, s$, and let $V$ be a space of $r$-vector functions $v(t)=\left(v^{1}, \ldots, v^{r}\right), t \in G$, whose components $v^{j}$ are $L_{p_{j}}$-integrable in $G, p_{j} \geqslant 1, j=1, \ldots, r$. Thus, $Y \subset L^{\prime}, V \subset L^{\prime \prime}$, where $L^{\prime}=\prod_{i=1}^{s} L_{p_{i}}(G), L^{\prime \prime}=\prod_{j=1}^{r} L_{p_{j}}(G)$. We shall take in $Y$ and $V$ the usual norms

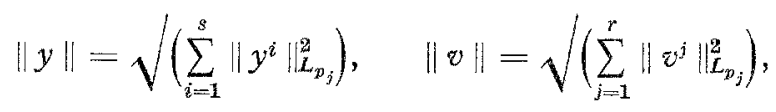

or equivalent ones, and we shall denote them also by $\|y\|_{p}$, or $\|y\|_{L_{p}}$.

Let $T$ be the space, or set, of all $m$-vector functions $u(t)=\left(u^{1}, \ldots, u^{m}\right)$, $t \in G$, whose components $u^{i}$ are measurable in $G$.

Let $S$ be a Banach space of elements $x$ and norm $\|x\|$, and let

$$
\mathscr{U}: S \rightarrow Y, \quad \mathscr{L}: S \rightarrow V
$$


be two given operators transforming every element $x \in S$ into elements $y=\mathscr{U} x \in Y, v=\mathscr{L} x \in V, y(t)=\left(y^{1}, \ldots, y^{s}\right), v(t)=\left(v^{1}, \ldots, v^{r}\right), t \in G$. Concerning $\mathscr{U}$ and $\mathscr{L}$, we shall assume that $(H)$ if $x, x_{k}, k=1,2, \ldots$, are elements of $S$ and $x_{k} \rightarrow x$ weakly in $S$ and $y_{k}=\mathscr{U} x_{k}, v_{k}=\mathscr{L} x_{k}, y=\mathscr{U} x, v=\mathscr{L} x$, then $y_{k} \rightarrow y$ strongly in $Y$, and $v_{k} \rightarrow v$ weakly in $V$.

For every $t \in \operatorname{cl} G=G \cup \partial G$, let $A(t)$ denote a nonempty subset of the $y$ space $E_{s}$, and let $A \subset E_{\nu+\delta}$ be the set of all $(t, y)$ with $t \in \operatorname{cl} G, y \in A(t)$. For every $(t, y) \in A$, let $U(t, y)$ denote a nonempty subset of the $u$-space $E_{m}$, and let $M \subset E_{v+s+m}$ be the set of all $(t, y, u)$ with $(t, y) \in A, u \in U(t, y)$. Let $f(t, y, u)=$ $\left(f_{1}, \ldots, f_{r}\right)$ be an $r$-vector function defined on $M$. We shall say that a pair $x, u$, $x \in S, u \in T$, is admissible provided the following conditions are satisfied: (a) $x \in S$; (b) $u \in T$, that is, $u(t)=\left(u^{1}, \ldots, u^{m}\right), t \in G$, with $u^{j}$ measurable in $G$, $j=1, \ldots, m$; (c) $y=\mathscr{U} x \in Y$, that is, $y(t)=\left(y^{1}, \ldots, y^{s}\right), t \in G$, with $y^{i} \in L_{p_{i}}(G)$, $i=1, \ldots, s ;$ (d) $v=\mathscr{L} x \in V$, that is, $v(t)=\left(v^{1}, \ldots, v^{r}\right), t \in G$, with $v^{j} \in L_{p_{j}}(G)$, $j=1, \ldots, r$; (e) $(t, y(t)) \in A$ a.e. in $G$; (f) $u(t) \in U(t, y(t))$ a.e. in $G$; and (g) $v(t)=f(t, y(t), u(t))$ a.e. in $G$. Requirement (g) can be written in the form

$$
(\mathscr{L} x)(t)=f(t,(\mathscr{U} x)(t), u(t)) \quad \text { a.e. in } G,
$$

an abstract functional equation. Whenever $S$ is a space of vector functions $x(t)$ on $G$ and $\mathscr{L}$ and $\mathscr{U}$ are differential operators, then (2) is a differential equation or system in $G$.

Remark 2.1. It will be enough to require that hypothesis $(H)$ is satisfied for sequences of elements $x$ which actually occur in the closed classes $\Omega$ of admissible pairs $x, u$ of the existence theorems in Sections 7 and 8 .

\section{Particular Case}

In the following situation, which is often encountered, less than $(H)$ is demanded. Let $X$ be a Banach space of elements $x$ and norm $\|x\|$, let $X_{o}$ be a linear subspace of $X$, let $\mathscr{U}: X_{o} \rightarrow Y, \mathscr{L}: X_{o} \rightarrow V$ be linear operators, and let $S$ be the completion of $X_{o}$ by means of the norm

$$
\|x\|=\sqrt{ }\left(\|x\|^{2}+\|\mathscr{U} x\|^{2}+\|\mathscr{L} x\|^{2}\right) .
$$

Then $S$ is a Banach space with norm $\|x\|$. Now, to each element $x \in S$ there corresponds a unique element $x_{o} \in X$, which we may denote simply by $x$, a vector function $y(t)=\left(y_{0}{ }^{1}, \ldots, y_{0}{ }^{s}\right), t \in G$, with $y^{i} \in L_{p_{i}}(G)$, a vector function $v_{o}(t)=\left(v_{0}^{1}, \ldots, v_{o}^{r}\right), t \in G$, with $v^{j} \in L_{p_{j}}(G)$, and sequences $\left[x_{k}\right]$ of elements $x_{k} \in X_{o}, \quad k=1,2, \ldots$, such that, if $y_{k}=\left(y_{k}{ }^{1}, \ldots, y_{k}{ }^{s}\right)=\mathscr{U} x_{k}, v_{k}=$ 
$\left(v_{k}{ }^{1}, \ldots, v_{k}{ }^{r}\right)=\mathscr{L} x_{k}, k=1,2, \ldots$, then $\left\|x_{k}-x\right\| \rightarrow 0,\left\|y_{k}{ }^{i}-y_{o}{ }^{i}\right\|_{p_{i}} \rightarrow 0$, $i=1, \ldots, s,\left\|v_{k}{ }^{j}-v_{o}{ }^{j}\right\|_{p_{j}} \rightarrow 0, j=1, \ldots, r$, as $k \rightarrow \infty$. We define $\mathscr{U}: S \rightarrow Y$, $\mathscr{L}: S \rightarrow V$ by taking $\mathscr{U} x=y_{o}, \mathscr{L} x=v_{0}$. It is known that $\mathscr{U}$ and $\mathscr{L}$ are uniquely defined and are linear operators from the Banach space $S$ into $Y$ and $V$, respectively.

Let $X^{*}$ be the topological dual of $X$, and let $\left(x, x^{*}\right)$ denote the application of $x^{*} \in X^{*}$ to $x \in X$. A sequence $\left[x_{k}\right]$ of elements $x_{k} \in S, k=1,2, \ldots$, converges weakly in $S$ to an element $x \in S$, or $x_{k} \rightarrow x$ weakly in $S$ as $k \rightarrow \infty$, provided the following conditions are satisfied: $\left(x_{k}, x^{*}\right) \rightarrow\left(x, x^{*}\right)$ for every $x^{*} \in X^{*}$; $\int_{G} y_{k}{ }^{i} \varphi d t \rightarrow \int_{G} y^{i} \varphi d t$ for every $\varphi \in L_{q_{i}}(G), q_{i}^{-1}+p_{i}^{-1}=1, i=1, \ldots, s$; and $\int_{G} v_{k}{ }^{j} \psi d t \rightarrow \int_{G} v^{j} \psi d t$ for every $\psi \in L_{q_{j}}(G), q_{j}^{-1}+p_{j}^{-1}=1, j=1, \ldots, r$. Here, $y_{\tilde{k}}=\left(y_{k}{ }^{1}, \ldots, y_{k}{ }^{s}\right)=\mathscr{U} x_{k}, v_{k}=\left(v_{k}{ }^{1}, \ldots, v_{k}^{r}\right) \in \mathscr{L} x_{k}, y=\left(y^{1}, \ldots, y^{s}\right)=\mathscr{U} x$, $v=\left(v^{1}, \ldots, v^{r}\right)=\mathscr{L}_{x}$.

In this situation, $x_{k} \rightarrow x$ weakly in $S$ certainly implies that $y_{k} \rightarrow y$ weakly in $Y$ and $v_{k} \rightarrow v$ weakly in $V$. The part of $(H)$ concerning $\mathscr{L}$ is thus trivial, and all we have to require is that $\left(H_{o}\right)$ if $x_{k} \rightarrow x$ weakly in $S$ as $k \rightarrow \infty$, then $y_{k} \rightarrow y$ strongly in $Y$ as $k \rightarrow \infty$, where $y_{k}=\mathscr{U} x_{k}, y=\mathscr{U} x, k=1,2, \ldots$.

\section{Orientor Fields and First Closure Theorem}

The abstract functional equation (2) can be written in terms of an orientor field. Indeed, for every $(t, y) \in A$, let $Q(t, y) \subset E_{r}$ denote the set

$$
Q(t, y)=f(t, y, U(t, y))=\left[z \in E_{r} \mid z=f(t, y, u), u \in U(t, y)\right] .
$$

Then, if $x, u$ is any admissible pair, then obviously $v(t) \in Q(t, y(t))$ a.e. in $G$, where $v=\mathscr{L} x, y=\mathscr{U} x$, or

$$
(\mathscr{L} x)(t) \in Q(t,(\mathscr{U} x)(t)) \quad \text { a.e. in } \mathrm{G} \text {. }
$$

This is the present abstract form of an orientor field equation.

If $G, A, S, Y, V, \mathscr{L}, \mathscr{U}$ are assigned as in Section 2, and we also assign for every $(t, y) \in A$ an arbitrary set $Q(t, y) \subset E_{r}$, then we may consider the corresponding director field relation in the form (5). We shall say then that an element $x \in S$ is admissible provided the following conditions are satisfied: $\left(\mathrm{a}^{\prime}\right) x \in S ;\left(\mathrm{b}^{\prime}\right) y=\mathscr{U} x \in Y, y(t)=\left(y^{1}, \ldots, y^{s}\right), t \in G, y^{i} \in L_{p_{i}}(G), i=1, \ldots, s ;$ $\left(\mathrm{c}^{\prime}\right) \quad v=\mathscr{L} x \in V, \quad v(t)=\left(v^{1}, \ldots, v^{r}\right), \quad t \in G, \quad v^{j} \in L_{p_{i}}(G), \quad j=1, \ldots, r ;\left(\mathrm{d}^{\prime}\right)$ $(t, y(t)) \in A$ a.e. in $G$; and $\left(\mathrm{e}^{\prime}\right) v(t) \in Q(t, y(t))$ a.e. in $G$, that is, relation (5) is satisfied.

If $x, y$ is any admissible pair, that is, $x, u$ satisfies (a) $-(\mathrm{g})$ of Section 2 and $Q(t, y)$ is defined by (4), then certainly $x \in S$ satisfies $\left(\mathrm{a}^{\prime}\right)-\left(\mathrm{e}^{\prime}\right), x$ is admis- 
sible and satisfies the director field relation (5). Conversely, if we know that $A$ and $M$ are closed, that $f$ is continuous on $M$, and that $Q(t, y)$ is defined by (4), then for every element $x \in S$ satisfying $\left(\mathrm{a}^{\prime}\right)-\left(\mathrm{e}^{\prime}\right)$ above, that is, for every solution of the director field relation (5), we can associate an element $u \in T$ such that $x, u$ is an admissible pair, that is, $x, u$ satisfies (a)-(g) of Section 2. The proof is based on the implicit function theorem (see, for example, Ref. 13).

Finally, we shall need below the property $(Q)$ for closed convex sets which we have used in Refs. 1-4 and 14-15. This concept is a variant for closed convex sets of Kuratowski's definition of upper semicontinuity of sets. A great many criteria for property $(Q)$ of the sets we shall deal with are known, and references will be given (Section 6 ).

For $(\bar{t}, \bar{y}) \in A$ and $\delta>0$, let $N_{\delta}(\bar{t}, \bar{y})$ denote the $\delta$-neighborhood of $(\bar{t}, \bar{y})$ in $A$, that is, the set of all $(t, y) \in A$ at a distance $\leqslant \delta$ from $(\bar{t}, \bar{y})$. For $(\bar{t}, \bar{y}) \in A$ and $\delta>0$, let $Q(\bar{t}, \bar{y} ; \delta)$ denote the union of all $Q(t, y)$ with $(t, y) \in N_{\delta}(\bar{t}, \bar{y})$. We say that the sets $Q(t, y)$ satisfy property $(Q)$ at the point $(\bar{t}, \bar{y}) \in A$ provided $Q(\bar{t}, \bar{y})=\bigcap_{\delta} \mathrm{cl} \operatorname{co} Q(\bar{t}, \bar{y}: \delta)$, or

$$
Q(\bar{t}, \bar{y})=\bigcap_{\delta} \operatorname{clco} \bigcup_{(t, y) \in N_{\delta}(\bar{z}, \bar{y})} Q(t, y) \text {. }
$$

We say that the sets $Q(t, y)$ satisfy property $(Q)$ in $A$ provided these sets satisfy the property above at every point $(t, y) \in A$. Sets $Q(t, y)$ satisfying property $(Q)$ are necessarily closed and convex.

Theorem 4.1 (First Closure Theorem). Let $G \subset E_{\nu}$ be open and bounded, and $A \subset E_{v+s}$ closed, let $S, Y, V, \mathscr{U}, \mathscr{L}$ as in Section 2; and, for every $(t, y) \in A$, let $Q(t, y)$ be a given subset of $E_{r}$ which we assume to be convex, closed, and to satisfy property $(Q)$ at every point of $A$ (with exception perhaps of a set of points whose $t$-coordinate lies in a set of measure zero on the $t$-space $E_{v}$ ).

Let $x, x_{k}, k=1,2, \ldots$, be elements of $S$, such that, if $y_{k}=\mathscr{U} x_{k}$, $v_{k}=\mathscr{L}_{x_{k}}, y=\mathscr{U} x, v=\mathscr{L} x$, then $x_{k} \rightarrow x$ weakly in $S, y_{k} \rightarrow y$ strongly in $Y$, $v_{k} \rightarrow v$ weakly in $V$ as $k \rightarrow \infty$. If all elements $x_{k}$ are admissible [that is, all $x_{k}$ satisfy $\left.\left(\mathrm{a}^{\prime}\right)-\left(\mathrm{e}^{\prime}\right)\right]$, then $x$ also is admissible.

This statement is a particular case of the following closure theorem.

\section{Second Closure Theorem}

Let $I_{o}$ be an interval of the $t$-space $E_{\nu}$ containing cl $G=G \cup \partial G$, where $G$ is a given bounded open subset of $E_{v}$. It is not restrictive to assume $I_{o}=[0, b]$, or $[0, \ldots, 0, b, \ldots, b]$, for some $b>0$. For every $t \in I_{o}, t=\left(t^{1}, \ldots, t^{\nu}\right)$, let $[0, t]$ denote the interval $\left[0, \ldots, 0, t^{1}, \ldots, t^{\nu}\right]$, or $0 \leqslant \tau^{i} \leqslant t^{i}, i=1, \ldots, \nu$. Let $Z$ be 
the space or set of all functions $z(t)=\left(z^{1}, \ldots, z^{\sigma}\right), t \in I$, which can be written in the form $z(t)=\int_{0}^{t} \psi(\tau) d \tau, t \in I_{o}$, where $d \tau=d \tau^{1} \cdots d \tau^{v}, \psi(t)=\left(\psi^{1}, \ldots, \psi^{v}\right)$, $t \in I_{o}$, and each component $\psi^{i}$ is $L$-integrable in $I_{o}$ and zero in $I_{o}-G$. Note that the elements $z(t)=\left(z^{1}, \ldots, z^{o}\right), t \in I_{o}$, of $Z$ certainly have the following properties: (1) each $z^{i}(t), t \in I_{o}$, is continuous on $I_{o}$ with values $z^{i}(t)=0$ whenever $t=\left(t^{1}, \ldots, t^{v}\right) \in I_{o}, t^{1} t^{2} \cdots t^{v}=0$; and (2) $z^{i}$ admits the generalized derivative $D^{\alpha_{0}} z^{i}$ of order $\alpha_{o}=(1,1, \ldots .1),\left|\alpha_{o}\right|=\nu$, and $D^{\alpha_{0}} z^{i}=\psi^{i}$ is $L$ integrable in $I_{o}$, and zero in $I_{o}-G$ (a.e.).

Let $G, A, U(t, y), M$ as in Section 2, let $N=r+\sigma$, and $f(t, y, u)=$ $\left(f, f_{o}\right)=\left(f_{1}, \ldots, f_{r}, f_{r+1}, \ldots, f_{r+\sigma}\right)$ be an $N$-vector function defined on $M$. Let $\widetilde{Q}(t, y) \subset E_{N}$ be the set defined by

$$
\widetilde{Q}(t, y)=\tilde{f}(t, y, U(t, y))=\left[\tilde{z} \in E_{N} \mid \tilde{z}=\tilde{f}(t, y, u), u \in U(t, y)\right] .
$$

Let $S, Y, V, T, \mathscr{U}, \mathscr{L}$ be defined as in Section 2. Instead of pairs $x, u$, we shall consider here systems $x, z, u$ with $x \in S, z \in Z, u \in T$. We shall say that a system $x, z, u$ is admissible provided all requirements (a)-(g) of Section 2 are satisfied and, in addition also (h) $z \in Z$ and (i) $D^{\alpha_{0}} z(t)=f_{o}(t, y(t), u(t))$ a.e. in $G, \alpha_{o}=(1,1, \ldots, 1)$. Thus, (g) and (i) together represent the increased functional system

$$
(\mathscr{L} x)(t)=f(t,(\mathscr{U} x)(t), u(t)), \quad\left(D^{\alpha_{o}} z\right)(t)=f_{o}(t,(\mathscr{U} x)(t), u(t)) \quad \text { a.e. in } G .
$$

As in Section 4, the system (7) can be written in the form of the orientor field relation

$$
\left((\mathscr{L} x)(t),\left(D^{\alpha_{o}} \mathcal{Z}\right)(t)\right) \in \widetilde{Q}(t,(\mathscr{U} x)(t)) \quad \text { a.e. in } G,
$$

where the first member is an $N$-vector, the second member is a subset of $E_{N}$, and $N=r+\sigma$.

Given $G$ and $A$ and, for any $(t, y) \in A$, a set $\tilde{Q}(t, y) \subset E_{N}$, we may consider the orientor field relation (8), with $S, Y, V, \mathscr{L}, \mathscr{U}$ defined as in Section 2. Then, a solution $x, z$ of this orientor field is now a pair $x, z$, satisfying $\left(\mathrm{a}^{\prime}\right)-\left(\mathrm{d}^{\prime}\right)$ of Section 4 and, in addition, also $\left(\mathrm{f}^{\prime}\right) z \in Z$ and $\left(\mathrm{g}^{\prime}\right)\left(v(t), D^{\alpha_{0}} z(t)\right) \in \widetilde{Q}(t, y(t))$ a.e. in $G$, which is again relation (8).

We have just proved that, for any system $x, z, u$ satisfying (a)-(i), the pair $x, z$ satisfies $\left(\mathrm{a}^{\prime}\right)-\left(\mathrm{d}^{\prime}\right),\left(\mathrm{f}^{\prime}\right),\left(\mathrm{g}^{\prime}\right)$, that is, the orientor field relation $(8)$ with the sets $\widetilde{Q}(t, y)$ defined by (6). Conversely, if $A$ and $M$ are closed, $\tilde{f}$ continuous on $M$, and the sets $\widetilde{Q}(t, y)$ are defined by (6), then for any pair $x, z$ satisfying $\left(a^{\prime}\right)-\left(d^{\prime}\right),\left(f^{\prime}\right),\left(g^{\prime}\right)$, that is, for any solution of the orientor field equation $(8)$, there is some element $u \in T$ such that $x, z, u$ satisfies all requirements (a)-(i), that is, $x, z, u$ is a solution of the increased functional system (7). 
We shall consider the countable set $\{\rho\}$ of all rationals, the countable system $\left\{t_{\rho}\right\}$ of all points $t=\left(t^{1}, \ldots, t^{v}\right)$ with $t^{j}=\rho_{j} b, \rho_{j} \in\{\rho\}, j=1, \ldots, \nu$, and the countable system $\{I\}$ of all intervals $I=\left[a_{1}, a_{2}\right] \subset I_{o}, a=\left(a_{1}^{1}, \ldots, a_{1}{ }^{\nu}\right)$, $a_{2}=\left(a_{2}{ }^{1}, \ldots, a_{2}{ }^{\nu}\right), a_{1}{ }^{i}<a_{2}{ }^{i}, i=1, \ldots, \nu$, with $a_{1}, a_{2} \in\left\{t_{\rho}\right\}$. For any given function $z(t), t \in I_{o}$, we shall consider the usual differences $\Delta z=\Delta_{I^{z}}$ of order $v$ relative to the $2^{v}$ vertices of $I$.

Let $G$ and $A$ as usual, $N=r+\sigma$; and, for every $(t, y) \in A$, let us consider a set $\mathscr{Q}(t, y) \subset E_{N}$ with the following properties: (1) there is a scalar $L$-integrable function $\psi(t) \geqslant 0, t \in G$, such that, if $z=\left(z^{1}, \ldots, z^{r}, z^{r+1}, \ldots, z^{r+o}\right) \in \varnothing(t, y)$, then $z^{i} \geqslant-\psi(t), i=r+1, \ldots, r+\sigma$; and (2) if

$$
\bar{z}=\left(\bar{z}^{1}, \ldots, \bar{z}^{2}, \bar{z}^{r+1}, \ldots, \bar{z}^{r+v}\right) \in \bar{Q}(t, y),
$$

then any other point $z=\left(\bar{z}^{1}, \ldots, \bar{z}^{r}, z^{r+1}, \ldots, z^{r+o}\right)$ with $z^{i} \geqslant \bar{z}^{i}, i=r+1, \ldots$, $r+\sigma$, belongs to $\bar{Q}(t, y)$.

Theorem 5.1 (Second Closure Theorem). Let $G$ be bounded and open, and $A \subset E_{\nu+8}$ closed as in Section 2, with $G \subset I_{0} \subset E_{\gamma}$, and let $S, Y, V, Z$, $\mathscr{L}, \mathscr{U}$ as in Sections 2 and 5. Let $N=r+\sigma$; and, for every $(t, y) \in A$, let $\tilde{Q}(t, y)$ be a subset of $E_{N}$ satisfying properties (1) and (2) above, and also convex, closed, and satisfying property $(Q)$ at every point of $A$ (with exception perhaps of a set of points whose $t$-coordinate lies in a set of measure zero on the $t$-space $E_{v}$ ). Let $x_{k}, z_{k}, k=1,2, \ldots, x_{k} \in S, z_{k} \in Z$, be pairs satisfying properties $\left(\mathrm{a}^{\prime}\right)-\left(\mathrm{d}^{\prime}\right),\left(\mathrm{f}^{\prime}\right),\left(\mathrm{g}^{\prime}\right)$, that is, admissible, and such that the following statements hold: $x_{k} \rightarrow x$ as $k \rightarrow \infty$ weakly toward an element $x \in S ; z_{k}(t) \rightarrow z(t)$ pointwise for all $t \in\left\{t_{\rho}\right\}, t \in I_{o} ; y_{k} \rightarrow y$ strongly in $Y$; and $v_{k} \rightarrow v$ weakly in $V$, where $y_{k}=\mathscr{U} x_{k}, v_{k}=\mathscr{L} x_{k}, y=\mathscr{U} x, v=\mathscr{L} x$, and $z(t)$ is defined only at the points $t \in\left\{t_{p}\right\}, t \in I_{o}$. Assume that there is a decomposition $z(t)=z_{o}(t)+s_{o}(t)$, $z_{o}(t)=\left(z_{0}^{1}, \ldots, z_{0}^{\sigma}\right), s_{0}(t)=\left(s_{0}^{1}, \ldots, s_{o}^{\sigma}\right)$, where $z_{o}$ is defined in the whole of $I_{o}$, $z_{0} \in Z$, and $s_{0}$ is singular. Then, the pair $x, z_{0}$ is admissible, that is, the pair $s, z_{0}$ satisfies $\left(\mathrm{a}^{\prime}\right)-\left(\mathrm{d}^{\prime}\right),\left(\mathrm{f}^{\prime}\right),\left(\mathrm{g}^{\prime}\right)$. The proof of this statement is essentially the same as the one in Ref. 2 , Section 3.3 .

\section{Lower Closure Theorem}

Here, $G, A, U(t, y), M$ are as in Section $2, f(t, y, u)=\left(f_{1}, \ldots, f_{r}\right)$, $f_{o}(t, y, u)$ are functions defined on $M, f_{o}$ scalar, and $S, Y, V, T, \mathscr{U}, \mathscr{L}$ are as in Section 2. We consider the class of all pairs $x, u, x \in S, u \in T$, satisfying all requirements (a)-(g) of Section 2, and, in addition, the following further 
requirement: (h) $f_{o}(t,(\mathscr{U} x)(t), u(t))$ is $L$-integrable in $G$. These pairs $x, u$ shall be denoted here as admissible pairs. Thus, for every admissible pair $x, u$, the functional

$$
I[x, u]=\int_{G} f_{o}(t,(\mathscr{U} x)(t), u(t)) d t
$$

is defined, and the constraints and the functional equation

$$
\begin{gathered}
(t,(\mathscr{U} x)(t)) \in A, \quad u(t) \in U(t,(\mathscr{U} x)(t)), \\
(\mathscr{L} x)(t)=f(t,(\mathscr{U} x)(t), u(t)) \quad \text { a.e. in } G,
\end{gathered}
$$

are satisfied. We say that the functional (9) possesses the property of lower closure at an element $x \in S$ provided the following statement holds: if $x_{k}, u_{k}$, $k=1,2, \ldots, x_{k} \in S, u_{k} \in T$, are admissible pairs, that is, $x_{k}, u_{k}$ satisfy (a)-(h), and $x_{k} \rightarrow x$ as $k \rightarrow \infty$ weakly in $S$, and $\lim I\left[x_{k}, u_{k}\right]<+\infty$ as $k \rightarrow \infty$, then there is an element $u \in T$ such that the pair $x, u$ is admissible [that is, $x, u$ satisfies (a)-(h)], and

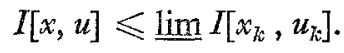

The usual concept of lower semicontinuity is a particular case of the concept of lower closure above. Indeed, assume that the data $G, A, U(t, y), M, f, f_{o}$, $S, Y, V, \mathscr{U}, \mathscr{L}$ are so arranged that $x$ determines $u$ uniquely. By this we mean, in symbols, that $\left(x, u_{1}\right),\left(x, u_{2}\right)$ admissible implies $u_{1}(t)=u_{2}(t)$ a.e in $G$, briefly $u_{1}=u_{2}$. Then, we can as well say that $x$ is admissible, we can denote $I[x, u]$ simply by $I[x]$, and (12) reduces to $I[x] \leqslant \varliminf I\left[x_{k}\right]$, the usual lower semicontinuity requirement, relatively to weak convergence in $S$. The situation that we have now depicted is very common. It occurs, for instance, with free problem, that is, problems concerning with the minimum of functionals of the form $I[x]=\int_{G} f_{o}(t, x(t),(\nabla x)(t)) d t$, where $x$ is a vector function in $G$ and $\nabla x$ the system of all first-order partial derivatives. Then, the problem can be written in the form (9)-(11) with $\nabla x=u$, that is, $x$ determines $u$ uniquely. The same situation occurs with the problems considered by Fichera (Refs. 8-10) concerning the minimum of functionals of the form $I[x]=$ $\int_{G} f_{o}(t,(\mathscr{U} x)(t),(\mathscr{L} x)(t)) d t$, where $x \in S, \mathscr{U}$ and $\mathscr{L}$ are as in Section 2. These problems can be written in the form (9)-(11) with $\mathscr{L} x=u$, that is, again, $x$ determines $u$ uniquely (see Sections 9.4 and 9.5 for details).

Theorem 6.1 (Lower Closure Theorem). Let $G, A, U(t, y), M$ as in Section 2, $G$ open and bounded, $G \subset I_{o} \subset E_{y}, A$ and $M$ closed, let $f(t, y, u)=$ 
$\left(f_{1}, \ldots, f_{r}\right), f_{o}(t, y, u)$ be continuous on $M, f_{o}$ scalar, and let us assume that the sets $\tilde{Q}(t, y)=\left[\tilde{z}=\left(z^{o}, z\right) \mid z^{o} \geqslant f_{o}(t, y, u), z=f(t, y, u), u \in U(t, y)\right] \subset E_{r+1} \gamma$ are convex, closed, and satisfy property $(Q)$ at every point of $A$ (with exception perhaps of a set of points whose $t$-coordinate lies in a set of measure zero on the $t$-space $E_{\gamma}$ ). Let us assume that $(\psi)$ for some scalar $L$-integrable function $\psi(t), t \in G, \psi \geqslant 0$, we have $f_{0}(t, y, u) \geqslant-\psi(t)$ for all $(t, y, u) \in M$. Let $S$, $Y, V, T, \mathscr{U}, \mathscr{L}$ be as in Section 2 with $\mathscr{L}, \mathscr{U}$ satisfying property $(H)$. Then, the functional (9) possesses the property of lower closure at every element $x \in S$. In other words, whenever $x \in S$ and there is a sequence of admissible pairs $x_{k}, u_{k}, k=1,2, \ldots$, with $x_{k} \rightarrow x$ as $k \rightarrow \infty$ weakly in $S$, and $\underline{\varliminf} I\left[x_{k}, u_{k}\right]<+\infty$, then there is some $u \in T$ such that $x, u$ is an admissible

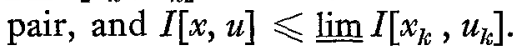

This statement is a corollary of the second closure theorem. Its proof is similar to the one in Ref. 2, Section 5.4. Condition $(\psi)$ in Theorem 6.1 can be replaced by the following weaker assumption $\left(\psi^{*}\right)$ : for every point $\bar{t} \in \operatorname{cl} G$, there are a neighborhood $N_{\delta}(\bar{t})$ of $\bar{t}$ in cl $G$, an $L$-integrable function $\psi(t) \geqslant 0$, $t \in N_{\delta}(\bar{t})$, and a real $r$-vector $b=\left(b_{1}, \ldots, b_{r}\right)$ such that $f_{o}-b \cdot f \geqslant-\psi$ in $N_{\delta}(\bar{t})$, that is,

$$
f_{o}(t, y, u)-\sum_{j=1}^{r} b_{j} f_{j}(t, y, u) \geqslant-\psi(t) \quad \text { for all }(t, y, u) \in M \quad \text { with } \quad t \in N_{\delta}(\bar{t}) .
$$

A proof of this extension of Theorem 6.1 is given in Ref. 16 for $\nu=1$.

By a well known remark by Goodman, the hypothesis in Theorem 6.1 that the functions $f_{o}, f$ are continuous on $M$ can be replaced by the weaker assumption that $f_{o}, f$ are continuous in $x, u$ for every $t$ and measurable in $t$ for every $x, u$. The proofs are essentially the same. Also, the assumption concerning the sets $\Phi(t, y)$ satisfying property $(Q)$ in Theorem 6.1 (as well as in Theorems 4.1 and 5.1) can be replaced by the following weaker assumption: there is a countable decomposition of $G$ into disjoint measurable sets $H_{\lambda}$, $\lambda=1,2, \ldots$, such that, if $A_{\lambda}$ denotes the set

$$
A_{\lambda}=\left[(t, y) \mid(t, y) \in A, t \in H_{\lambda}\right] \subset E_{\gamma+s},
$$

then the sets $\widetilde{Q}(t, y)$ satisfy property $(Q)$ in $A_{\lambda}$ for almost every $t, \lambda=1,2, \ldots$.

Whenever $x$ determines $u$ uniquely (see above), Theorem 6.1 (and its extensions) reduces to a sufficient condition for lower semicontinuity. As such, it contains as particular cases the lower semicontinuity theorems with respect to weak convergence due to Morrey for free problems (see Section 9.4 below) and Fichera for his class of problems (see Section 9.5 below). In both 
cases, $r=m, f=u$, hence $\mathscr{L}_{x}=u$, and the functional can simply be written $I[x]$.

Corollary 6.1. Under the hypotheses of Theorem 6.1, with $r=m$, $f=u$, hence $\mathscr{L} x=u$, and

$$
\left.\widetilde{Q}(t, y)=\left[\left(z^{o}, u\right)\right] z^{o} \geqslant f_{o}(t, y, u), u \in U(t, y)\right] \subset E_{r+1},
$$

if $x_{k} \rightarrow x$ weakly in $S$, and $\varliminf_{1} I\left[x_{k}\right]<\infty$, then $I[x] \leqslant \varliminf I\left[x_{k}\right]$.

Indeed, the weak convergence $x_{k} \rightarrow x$ implies $\mathscr{U} x_{k} \rightarrow \mathscr{U} x$ strongly, $\mathscr{L} x_{k} \rightarrow \mathscr{L} x$ weakly. Hence, $\mathscr{L} x_{k}=u_{k}, k=1,2, \ldots$, implies $u_{k} \rightarrow u_{0}$ weakly for some element $u_{o}$, and $u_{o}=\mathscr{L} x$. On the other hand, the element $u$ guaranteed by Theorem 6.1 also satisfies $u=\mathscr{L} x$, and thus $u=u_{o}$, and $I[x] \leqslant \underline{\lim } I\left[x_{k}\right]$.

For a further analysis of the concepts of lower closure and lower semicontinuity, see Ref. 16.

In Refs. 15 and 17, we have given criteria for property $(Q)$ of the sets $\widetilde{Q}(t, y)$. We mention here that a function $g(t, y, u)$ is said to be of slower growth than $f_{o}$ with respect to $u$ in a set $A_{0} \subset A$ provided, given $\epsilon>0$, there is some $N \geqslant 0$ (depending on $\left.g, f_{o}, \epsilon, A_{o}\right)$ such that $(t, y, u) \in M,|u| \geqslant N$ implies $|g(t, y, u)| \leqslant \epsilon f_{o}(t, y, u)$. We proved in Ref. 15 that, if 1 and $f$ are of slower growth than $f_{o}$ with respect to $u$ in a neighborhood $N_{\delta}(\bar{t}, \bar{y})$ of $(\bar{t}, \bar{y}) \in A$, then the sets $\mathscr{Q}(t, y)$, if convex, certainly are closed and satisfy property $(Q)$ at $(\bar{t}, \bar{y})$. Other criteria for property $(Q)$ of the sets $\tilde{Q}(t, y)$ have been given in Refs. 16-17 in terms of the supporting planes of the convex sets $\widetilde{Q}(t, y)$, and in this respect property $(Q)$ corresponds to the property of seminormality introduced by Tonelli and McShane for free problems (see Ref. 16-17 for details). Other criteria for property $(Q)$ have been given by Olech (Refs. 18-19). Nevertheless, Olech's lower closure and existence theorems contain conditions which are rather demanding when compared with our condition $\left(\psi^{*}\right)$. For comparison and examples, see Ref. 16.

Note that, in Theorem 6.1 (as well as in the closure theorems of Sections 4 and 5), no topology has been chosen in $T$, the set of all measurable vector functions $u(t)=\left(u^{1}, \ldots, u^{m}\right), t \in G$. Hence, the question of what happens if the vector functions $u_{k}$ are known to converge toward some function $u_{o}$ does not arise in this situation. Nevertheless, if we assume that the functions $u_{k}$ are in $\left(L_{p}(G)\right)^{m}$ for some $p \geqslant 1$ and that the functions $u_{k}$ converge weakly in $\left(L_{p}(G)\right)^{m}$ toward some element $u_{o}=\left(u_{o}^{1}, \ldots, u_{o}{ }^{m}\right)$ of this space (that is, $u_{k}^{i} \rightarrow u_{o}^{i}$ weakly in $L_{p}(G)$ as $\left.k \rightarrow \infty, i=1, \ldots, m\right)$, then we may ask whether the pair $x, u_{o}$ is admissible and whether the relation $I\left[x, u_{o}\right] \leqslant \underline{\lim } I\left[x_{k}, u_{k}\right]$ holds. We have already seen that, for free problems, the answer is affirmative 
(Corollary 6.1). The following Corollary 6.2 shows that, even in the general case, the answer is affirmative under assumptions. We shall assume precisely that $f$ is linear in $u$, that is, $f(t, y, u)=B(t, y) u+C(t, y), B, C$ matrices of the types $r \times m, r \times 1$ respectively, with entries continuous in $A$. This is certainly the case for free problems where $r=m$ and $f=u$. Instead of the sets $\not{Q}$, we shall consider the sets

$$
\begin{aligned}
\bar{Q}^{*}(t, y) & =\left[\left(z^{o}, z\right) \mid z^{o} \geqslant f_{0}(t, y, u), z=u, u \in U(t, y)\right] \\
& =\left[\left(z^{o}, u\right) \mid z^{o} \geqslant f_{0}(t, y, u), u \in U(t, y)\right] \subset E_{m+1} .
\end{aligned}
$$

Corollary 6.2. Under the conditions of Theorem 6.1, with

$$
f=B(t, y) u+C(t, y)
$$

and the sets $\mathscr{Q}^{*}$ replacing the sets $\Phi$, if the functions $u_{k}$ are in $\left(L_{p}(G)\right)^{m}$, $p>1$, and $u_{k} \rightarrow u_{o}$ weakly in $L_{p}$ as $k \rightarrow \infty$, then the pair $x, u_{o}$ is admissible, and $I\left[x, u_{o}\right] \leqslant \underline{l} I\left[x_{k}, u_{k}\right]$. The same statement holds for $p=1$ provided in addition we know that the functions $u_{k}$ are equiabsolutely integrable in $G$.

This corollary was proved in Ref. 16 for $\nu=1$. The proof for any $\nu$ is essentially the same.

Note that, in Corollary 6.2 , for $p=1$ the functions $u_{k}$ are certainly equiabsolutely integrable in $G$ under suitable growth conditions. For instance, a suitable growth condition is the following: $\left(\epsilon_{0}\right)$ for each $\epsilon>0$, there is some integrable function $\psi_{\epsilon}(t) \geqslant 0, t \in G$, such that $|u| \leqslant \psi_{\epsilon}(t)+\epsilon f_{o}(t, y, u)$ for all $(t, y, u) \in M$. An analogous growth condition was used in Ref. 15 for a different purpose and will be mentioned in Section 9.4 below.

In Corollary 6.2, the specific hypotheses cannot be omitted in general, as examples show (see Ref. 16 for $\nu=1$ ).

\section{Existence Theorems for Abstract Multidimensiona1 Lagrange Problems}

We shall use here the same notations as in Section 6, and assume that the general hypotheses of lower closure theorem 6.1 (or of one of its extensions) are satisfied. Thus, an admissible pair $x, u$ is a pair of elements $x \in S, u \in T$, satisfying (a)-(g) of Section 2 and (h) of Section 6 . We shall consider classes $\Omega$ of such admissible pairs, which are closed in the following sense: if $x \in S$, if $x_{k}, u_{k}, k=1,2, \ldots$, are admissible pairs and belong to $\Omega$, if $x_{k} \rightarrow x$ weakly 
in $S$, and $\underline{\lim } I\left[x_{k}, u_{k}\right]<+\infty$ as $k \rightarrow \infty$, then at least one of the elements $u \in T$ guaranteed by the lower closure theorem of Section 6 is such that $x, u$ belongs to $\Omega$ (besides $x, u$ being an admissible pair, and $I[x, u] \leqslant \underline{\lim } I\left[x_{k}, u_{k}\right]$ as stated in lower closure theorem). Obviously, the class of all admissible pairs is certainly closed in this sense, under the same general assumptions of the lower closure theorem of Section 6 (or of one of its extensions).

Given a family $\Omega=\{(x, u)\}$ of admissible pairs $x, u, x \in S, u \in T$, we shall also consider the corresponding class $\{x\}_{\Omega}$ of the elements $x$ in $\Omega$; in symbols, $\{x\}_{\Omega}=\{x \in S \mid(x, u) \in \Omega$ for some $u \in T\}$. We shall consider closed classes $\Omega$ of admissible pairs $x, u$ such that the corresponding set $\{x\}_{\Omega}$ is weakly sequentially compact.

Theorem 7.1 (Existence Theorem for Abstract Multidimensional Lagrange Problems). Let $G, A, U(t, y), M$ be as in Section 2 with $G$ bounded and open, $A, M$ closed, let $f(t, y, u)=\left(f_{1}, \ldots, f_{r}\right), f_{o}(t, y, u)$ be continuous on $M, f_{o}$ scalar, and let $S, Y, V, T, \mathscr{U}, \mathscr{L}$ as in Section 2 , with $\mathscr{U}, \mathscr{L}$ satisfying property $(H)$. Let us assume that the sets

$$
\tilde{Q}(t, y)=\left[\tilde{z}=\left(z^{o}, z\right) \mid z^{o} \geqslant f_{o}(t, y, u), z=f(t, y, u), u \in U(t, y)\right]
$$

are convex, closed, and satisfy property $(Q)$ at every point of $A$ (with exception perhaps of a set of points whose $t$-coordinate lies in a set of measure zero on the $t$-space $\left.E_{v}\right)$. Let us assume that $(\psi)$ for some scalar $L$-integrabie function $\psi(t) \geqslant 0, t \in G$, we have $f_{o}(t, y, u) \geqslant-\psi(t)$ for all $(t, y, u) \in M$. Let $\Omega$ be a nonempty closed class of admissible pairs $x, u$ [that is, $x \in S, u \in T, x, u$ satisfying (a)-(g) of Section 2 and $(\mathrm{h})$ of Section 6], in particular, satisfying the constraints (10) and the functional relation (11), and let assume that the corresponding set $\{x\}_{\Omega}$ is weakly sequentially compact in $S$. Then, the functional (9) possesses an absolute minimum in $\Omega$.

The proof is the same as in (Ref. 2, Section 5.4).

Remark 7.1. The same operator $\mathscr{U}$ appears in (9)-(11). The case in which different operators $\mathscr{U}_{1}, \mathscr{U}_{2}, \mathscr{U}_{3}$ appear in (9), (10), (11), respectively, is only a particular case of the one under consideration. Indeed, if $y_{1}=\mathscr{U}_{1} x$, $y_{2}=\mathscr{U}_{2} x, y_{3}=\mathscr{U}_{3} x$, we may denote by $\mathscr{U}$ the unique operator $\mathscr{U} x=$ $\left(y_{1}, y_{2}, y_{3}\right)$, with the convention that $f_{0}$ depends only on $y_{1}, f$ depends only on $y_{3}$, and $A$ is a cylinder set $A=A_{o} \times E_{k}$, where $k$ is the dimension of the vector $\left(y_{1}, y_{3}\right)$. The existence theorem above holds without changes provided we assume that $\mathscr{U}_{1}, \mathscr{U}_{2}, \mathscr{U}_{3}$ satisfy axiom $(H)$, that is, $\mathscr{U}$ satisfies $(H)$. 
Remark 7.2. Extensions of Theorem 7.1 hold which are analogous to those mentioned for Theorem 6.1. First, the hypothesis $(\psi)$ can be replaced by the following weaker one: $\left(\psi^{*}\right)$ for every point $\bar{t} \in \mathrm{cl} \mathrm{G}$, there are a neighborhood $N_{\delta}(\bar{t})$ of $\bar{t}$ in cl $G$, a scalar $L$-integrable function $\psi(t) \geqslant 0, t \in N_{\delta}(\bar{t})$, and real numbers $b=\left(b_{1}, \ldots, b_{r}\right)$ such that $f_{o}-b \cdot f \geqslant-\psi$ for all $(t, y, u) \in M$ with $t \in N_{\delta}(\bar{t})$ (see Ref. 17). The hypothesis of continuity of $f$ and $f_{o}$ on $M$ can be replaced by the weaker one that $f$ and $f_{o}$ are continuous in $x, u$ for every $t$ and measurable in $t$ for every $x, u$. Also, the assumption concerning the sets $\widetilde{Q}(t, y)$ satisfying property $(Q)$ in Theorem 7.1 can be replaced by the following weaker hypothesis: there is a countable decomposition of $G$ into disjoint measurable sets $H_{\lambda}, \lambda=1,2, \ldots$, such that, if $A_{\lambda}$ denotes the set $A_{\lambda}=$ $\left[(t, y) \mid(t, y) \in A, t \in H_{\lambda}\right]$, then the sets $\mathscr{Q}(t, y)$ satisfy property $(Q)$ in $A_{\lambda}$ for almost every $t, \lambda=1,2, \ldots$.

As usual, conditions of the form $\left\|y^{i}\right\|_{p_{i}} \leqslant M_{i}$ for given constants $M_{i}$ guarantee the weak sequential compactness of the functions $y^{i}$ in $G$ whenever $p_{i}>1$. For $p_{i}=1$, the same condition together with suitable growth conditions can be used. For instance, the following condition has been used: $(\epsilon)$ given $\epsilon>0$ there is an integrable function $\psi_{\epsilon}(t) \geqslant 0, t \in G$, such that $\left|f_{i}(t, y, u)\right| \leqslant$ $\psi_{\epsilon}(t)+\epsilon f_{o}(t, y, u)$ (see Refs. 4 and 15).

Remark 7.3. The existence theorem above applies as well to generalized solutions (Gamkrelidze's chattering states). In the present context, we denote by generalized solution any system $(x, p, w)$ such that the following statements hold: $x \in S ; p=p(t)=\left(p_{1}, \ldots, p_{\mu}\right), t \in G, p_{j}(t)$ measurable in $G$, $p_{j}(t) \geqslant 0, \quad j=1, \ldots, \mu, \quad \sum_{j} p_{j}(t)=1 ; \quad w=w(t)=\left(u^{(1)}, \ldots, u^{(\mu)}\right), \quad u^{(j)} \in T$, $j=1, \ldots, \mu ; \quad y=\mathscr{U} x \in Y ; \quad v=\mathscr{L} x \in V ; \quad(t, y(t)) \in A$;

$$
\vartheta(t)=\sum_{j} p_{j}(t) f\left(t, y(t), u^{(j)}(t)\right) \quad \text { a.e. in } G ;
$$

and $\sum_{j} p_{j}(t) f_{o}\left(t, y(t), u^{(j)}(t)\right) L$-integrable in $G$ (see Sections 2 and 6). Thus, we are concerned with a system monitored by a functional equation of the form

$$
(\mathscr{L} x)(t)=\sum_{j=1}^{\mu} p_{j}(t) f\left(t,(\mathscr{U} x)(t), u^{(j)}(t)\right) \quad \text { a.e. in } G,
$$

with usual constraints

$$
(t,(\mathscr{U} x)(t)) \in A, \quad u^{(j)}(t) \in U(t,(\mathscr{U} x)(t)), \quad j=1, \ldots, \mu, \quad \text { a.e. in } G,
$$

and functional

$$
J[x, p, w]=\int_{G} \sum_{j=1}^{u} p_{j}(t) f_{o}\left(t,(\mathscr{U} x)(t), u^{(j)}(t)\right) d t .
$$


For any integer $\mu \geqslant r+2$, the corresponding sets $\tilde{Q}(t, y) \subset E_{r+1}$ are the sets $\tilde{R}(t, y)=\operatorname{co} \widetilde{Q}(t, y) \subset E_{r+1}$, which are necessarily convex. It is then assumed that $\mu$ above is the minimum integer for which this occurs. The existence theorem above holds for generalized solutions with the convexity requirement necessarily satisfied.

\section{Extensions}

Let us assume that the $m$ components $u=\left(u^{1}, \ldots, u^{m}\right)$ of the variable $u$ can be divided into two classes, say $u^{\prime}=\left(u^{1}, \ldots, u^{\alpha}\right)$ and $u^{\prime \prime}=\left(u^{\alpha+1}, \ldots, u^{m}\right)$, so that we can write $u=\left(u^{\prime}, u^{\prime \prime}\right)$, and that $U(t, y)=U^{\prime}(t, y) \times U^{\prime \prime}(t, y)$ for all $(t, y) \in A$ with $U^{\prime}(t, y) \in E_{\alpha}, U^{\prime \prime}(t, y) \in E_{m \rightarrow \alpha}, 0 \leqslant \alpha \leqslant m$. Let us restrict the class $T$ of Section 2 to a slightly smaller class $T=T^{\prime} \times T^{\prime \prime}$, where $T^{\prime}$ is the set of all measurable vector functions $u^{\prime}(t)=\left(u^{1}, \ldots, u^{\alpha}\right), t \in G$, and $T^{\prime \prime}$ is a Banach space of vector function $u^{\prime \prime}(t)=\left(u^{\alpha+1}, \ldots, u^{m}\right), t \in G$, with norm $\left\|u^{\prime \prime}\right\|$, say,

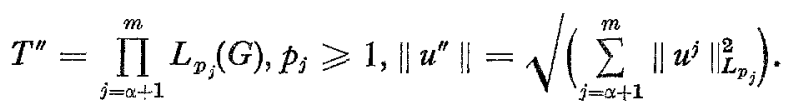

We shall denote by $M^{\prime}, M^{\prime \prime}$ the sets

$$
\begin{aligned}
M^{\prime} & =\left[\left(t, y, u^{\prime}\right) \mid(t, y) \in A, u^{\prime} \in U^{\prime}(t, y)\right] \subset E_{\gamma+s+\alpha}, \\
M^{\prime \prime} & =\left[\left(t, y, u^{\prime \prime}\right) \mid(t, y) \in A, u^{\prime \prime} \in U^{\prime \prime}(t, y)\right] \subset E_{\gamma+s+m-\alpha},
\end{aligned}
$$

so that

$$
M=\left[\left(t, y, u^{\prime}, u^{\prime \prime}\right) \mid(t, y) \in A, u^{\prime} \in U^{\prime}(t, y), u^{\prime \prime} \in U^{\prime \prime}(t, y)\right] \subset E_{\gamma+s+m}
$$

We shall assume that $f_{o}$ and $f$ are of the form

$$
\begin{gathered}
f_{o}\left(t, y, u^{\prime}, u^{\prime \prime}\right)=f_{o}^{\prime}\left(t, y, u^{\prime}\right)+f_{o}^{\prime \prime}\left(t, y, u^{\prime \prime}\right), \\
f\left(t, y, u^{\prime}, u^{\prime \prime}\right)=f^{\prime}\left(t, y, u^{\prime}\right)+f^{\prime \prime}\left(t, y, u^{\prime \prime}\right),
\end{gathered}
$$

where $f_{o}^{\prime}, f^{\prime}$ are defined on $M^{\prime}, f_{o}^{\prime \prime}$ is defined on $M^{\prime \prime}$, and $f^{\prime \prime}$ is linear in $u^{\prime \prime}$, or $f^{\prime \prime}\left(t, y, u^{\prime \prime}\right)=B(t, y) u^{\prime \prime}+C(t, y), B, C$ matrices of the types $r \times(m-\alpha)$, $r \times 1$ respectively, with entries defined on $A$.

From Section 2, we know that $V C L^{\prime \prime}=\prod_{j=1}^{r} L_{p_{j}}(G)$. We shall need below the following growth condition. We say that $f=\left(f_{1}, \ldots, f_{r}\right)$ and $f_{o}$ satisfy a growth condition $\left(\epsilon_{p}\right)$ provided: for every $j=1, \ldots, r$ with $p_{j}>1$, 
there are constants $a>0, b>0$ and a function $\psi \in L(G), \psi(t) \geqslant 0$, such that $\left|f_{j}\right|^{p_{j}} \leqslant a \psi(t)+b f_{o}$ everywhere in $M$; for $j=1, \ldots, r$ with $p_{j}=1$, then for every $\epsilon>0$ there is a function $\psi_{\epsilon} \in L(G), \psi_{\epsilon}(t) \geqslant 0$, such that $\left|f_{j}\right| \leqslant$ $\psi_{\epsilon}(t)+\epsilon f_{o}$. Note that, under such condition $\left(\epsilon_{p}\right)$, we have $f_{o} \geqslant-b^{-1} a \psi$, or $f_{o} \geqslant-\psi_{1}$, respectively, where $\psi, \psi_{1}$ are fixed nonnegative $L$-integrable functions in $G$. An analogous growth condition $\left(\epsilon_{p}\right)$ could be required for the pair $f^{\prime}, f_{o}^{\prime}$, or for the pair $f^{\prime \prime}, f_{o}^{\prime \prime}$.

We shall need the sets

$$
\begin{aligned}
\tilde{Q}^{\prime}(t, y) & =\left[\tilde{z}=\left(z^{o}, z\right) \mid z^{o} \geqslant f_{o}^{\prime}\left(t, y, u^{\prime}\right), z=f^{\prime}\left(t, y, u^{\prime}\right), u^{\prime} \in U^{\prime}(t, y)\right] \subset E_{r+1}, \\
\tilde{Q}^{*}(t, y) & =\left[\tilde{z}=\left(z^{o}, u^{\prime \prime}\right) \mid z^{o} \geqslant f_{o}^{\prime \prime}\left(t, y, u^{\prime \prime}\right), u^{\prime \prime} \in U^{\prime \prime}(t, y)\right] \subset E_{m-\alpha+1} .
\end{aligned}
$$

We shall assume that $\mathscr{U}: S \times T^{\prime \prime} \rightarrow Y, \mathscr{L}: S \times T^{\prime \prime} \rightarrow V$ are defined on $S \times T^{\prime \prime}$, that is, $y=\mathscr{U}\left(x, u^{\prime \prime}\right) \in Y, v=\mathscr{L}\left(x, u^{\prime \prime}\right) \in V$ for all $\left(x, u^{\prime \prime}\right) \in S \times T^{\prime \prime}$. Also, we shall denote by $V_{o}$ a space of real-valued functions $v_{o}(t), t \in G$, which are $L_{p_{o}}$-integrable in $G$ for some $p_{o} \geqslant 1$, or $V_{o} \subset L_{p_{o}}(G)$, and we shall take in $V_{o}$ the norm $\left\|v_{o}\right\|_{p_{o}}$. We shall then consider $\mathscr{L}_{o}\left(x, u^{\prime \prime}\right)$, or $\mathscr{L}_{o}: S \times T^{\prime \prime} \rightarrow V_{o}$, mapping each pair $\left(x, u^{\prime \prime}\right) \in S \times T^{\prime \prime}$ into an element $v_{o}=\mathscr{L}_{0}\left(x, u^{\prime \prime}\right) \in V_{o}$. Instead of axiom $(H)$, we shall now assume the following: $\left(H^{*}\right)$ if $x, x_{k} \in S$, $u^{\prime \prime}, u_{k}^{\prime \prime} \in T^{\prime \prime}, k=1,2, \ldots$, if $x_{k} \rightarrow x$ weakly in $S$ and $u_{k}^{\prime \prime} \rightarrow u^{\prime \prime}$ weakly in $T^{\prime \prime}$, then $y_{k} \rightarrow y$ strongly in $Y, v_{k} \rightarrow v$ weakly in $V, v_{o k} \rightarrow v_{o}$ weakly in $V_{o}$, where $y=\mathscr{U}\left(x, u^{\prime \prime}\right), v=\mathscr{L}\left(x, u^{\prime \prime}\right), v_{o}=\mathscr{L}_{o}\left(x, u^{\prime \prime}\right), y_{k}=\mathscr{U}\left(x_{k}, u_{k}^{\prime \prime}\right), v_{k}=\mathscr{L}\left(x_{k}, u_{k}^{\prime \prime}\right)$, $v_{o k}=\mathscr{L}_{o}\left(x_{k}, u_{k}^{\prime \prime}\right)$

In the present situation, we say that a pair $x, u(t), t \in G$, is admissible provided $x \in S, \quad u=\left(u^{\prime}, u^{\prime \prime}\right), \quad u \in T^{\prime}, u^{\prime \prime} \in T^{\prime \prime}, \quad y=\mathscr{U}\left(x, u^{\prime \prime}\right) \in Y, \quad v=$ $\mathscr{L}\left(x, u^{\prime \prime}\right) \in V, \quad(t, y(t)) \in A$ a.e. in $G, u^{\prime}(t) \in U^{\prime}(t, y(t)), u^{\prime \prime}(t) \in U^{\prime \prime}(t, y(t))$ a.e. in $G, v(t)=f^{\prime}\left(t, y(t), u^{\prime}(t)\right)+f^{\prime \prime}\left(t, y(t), u^{\prime \prime}(t)\right)$ a.e. in $G$, and $f_{o}^{\prime}\left(t, y(t), u^{\prime}(t)\right)+f_{0}^{\prime \prime}\left(t, y(t), u^{\prime \prime}(t)\right)$ is $L$-integrable in $G, v_{o}=\mathscr{L}\left(x, u^{\prime \prime}\right) \in V_{o}$.

We deal here with the problem of the minimum of a functional

$$
I\left[x, u^{\prime}, u^{\prime \prime}\right]=\int_{G}\left[f_{o}^{\prime}\left(t,\left(\mathscr{U}\left(x, u^{\prime \prime}\right)\right)(t), u^{\prime}(t)\right)+f_{0}^{\prime \prime}\left(t,\left(\mathscr{U}\left(x, u^{\prime \prime}\right)\right)(t), u^{\prime \prime}(t)\right)+\mathscr{L}_{0}\left(x, u^{\prime \prime}\right)(t)\right] d t
$$

in a class $Q$ of admissible pairs $x, u(t)=\left(u^{\prime}, u^{\prime \prime}\right), t \in G$. The constraints and functional relation are now of the forms

$$
\begin{gathered}
\left(\mathscr{L}\left(x, u^{\prime \prime}\right)\right)(t)=f^{\prime}\left(t,\left(\mathscr{U}\left(x, u^{\prime \prime}\right)\right)(t), u^{\prime}(t)\right)+f^{\prime \prime}\left(t,\left(\mathscr{U}\left(x, u^{\prime \prime}\right)\right)(t), u^{\prime \prime}(t)\right) \quad \text { a.e. in } G, \\
u^{\prime}(t) \in U^{\prime}\left(t,\left(\mathscr{U}\left(x, u^{\prime \prime}\right)\right)(t)\right), \quad u^{\prime \prime}(t) \in U^{\prime \prime}\left(t,\left(\mathscr{U}\left(x, u^{\prime \prime}\right)\right)(t)\right) \quad \text { a.e. in } G, \\
\left(t,\left(\mathscr{U}\left(x, u^{\prime \prime}\right)\right)(t)\right) \in A .
\end{gathered}
$$

We are now in a position to state the following extension of Corollary 6.2 . 
Corollary 8.1. Assume that a decomposition (13), holds with $f^{\prime \prime}$ linear in $u^{\prime \prime}$. Assume that $f_{o}^{\prime} \geqslant-\psi^{\prime}, f_{o}^{\prime \prime} \geqslant-\psi^{\prime \prime}$ for some function $\psi^{\prime}, \psi^{\prime \prime} \in L(G)$, $\psi^{\prime} \geqslant 0, \psi^{\prime \prime} \geqslant 0$. Assume that either the pair $f^{\prime}, f_{o}^{\prime}$, or the pair $f^{\prime \prime}, f_{o}^{\prime \prime}$, or both, satisfy a growth condition $\left(\epsilon_{p}\right)$. If $U(t, y)=U^{\prime}(t, y) \times U^{\prime \prime}(t, y)$ as above, if the sets $\widetilde{Q}^{\prime}(t, y)$ and $\Phi^{*}(t, y)$ are closed, convex, and satisfy property $(Q)$ in $A$ (or in any of the modes mentioned in Section 6), if the operators $\mathscr{L}, \mathscr{U}$, $\mathscr{L}_{0}$ as above satisfy axiom $\left(H^{*}\right)$, if $x_{k}, u_{k}, k=1,2, \ldots$, is a sequence of admissible pairs, with $x_{k} \in S, u_{k}=\left(u_{k}{ }^{\prime}, u_{k}^{\prime \prime}\right), u_{k}{ }^{\prime} \in T^{\prime}, u_{k}^{\prime \prime} \in T^{\prime \prime}$, and if $x_{k} \rightarrow x$ weakly in $S, u_{k}^{\prime \prime} \rightarrow u_{0}^{\prime \prime}$ weakly in $T^{\prime \prime}, \underline{\lim } I\left[x_{k}, u_{k}{ }^{\prime}, u_{k}^{\prime \prime}\right]<\infty$, then the element $u=\left(u^{\prime}, u^{\prime \prime}\right)$ of Theorem 6.1 can be so chosen that $u^{\prime \prime}=u_{0}^{\prime \prime}, x, u$ is admissible, and $I\left[x, u^{\prime}, u^{\prime \prime}\right] \leqslant \underline{\lim } I\left[x_{k}, u_{k}^{\prime}, u_{k}^{\prime \prime}\right]$.

The proof is the same as for Corollary 6.2 (see Ref. 16, where proofs are given for $\nu=1$ ). Note that Corollary $6.2 \mathrm{can}$ be obtained from Corollary 8.1 by taking $\alpha=0, u^{\prime \prime}=u, f^{\prime}=0, f_{o}{ }^{\prime}=0, f^{\prime \prime}=f, f_{o}^{\prime \prime}=f_{o}$, and by remarking that the pair $f^{\prime}=0, f_{0}^{\prime}=0$ satisfies trivially a growth condition $\left(\epsilon_{p}\right)$ and that the corresponding sets $Q^{\prime}$ trivially satisfy condition $(Q)$.

We shall say now that a class $\Omega$ of admissible pairs $x, u$ is closed provided the following statement holds: if $x \in S, u^{\prime \prime} \in T^{\prime \prime}$, if $x_{k}, u_{k}$ with $u_{k}=\left(u_{k}{ }^{\prime}, u_{k}^{\prime \prime}\right)$, $k=1,2, \ldots$, are admissible pairs and belong to $\Omega$, if $x_{k} \rightarrow x$ weakly in $S$ and $u_{k}^{\prime \prime} \rightarrow u^{\prime \prime}$ weakly in $T^{\prime \prime}$, and $\underline{\lim } I\left[x_{k}, u_{k}{ }^{\prime}, u_{k}^{\prime \prime}\right]<+\infty$ as $k \rightarrow \infty$, then at least one of the elements $u^{\prime} \in T^{\prime}$ guaranteed by the lower closure theorem is such that the pair $x, u$ with $u=\left(u^{\prime}, u^{\prime \prime}\right)$ belongs to $\Omega$. Finally, given any class $\Omega$ of admissible pairs $x, u$ with $u=\left(u^{\prime}, u^{\prime \prime}\right)$, we shall consider the set $\{x\}_{\Omega}=$ $\left\{x \mid x \in S,(x, u) \in \Omega\right.$ for some $\left.u=\left(u^{\prime}, u^{\prime \prime}\right) \in T^{\prime} \times T^{\prime \prime}\right\}$ and the set $\left\{u^{\prime \prime}\right\}_{\Omega}=$ $\left\{u^{\prime \prime} \mid u^{\prime \prime} \in T^{\prime \prime},\left(x, u^{\prime}, u^{\prime \prime}\right) \in \Omega\right.$ for some $\left.x \in S, u^{\prime} \in T^{\prime}\right\}$.

The existence theorem of Section 7 holds now under axiom $\left(H^{*}\right)$, under the hypotheses that the sets $\tilde{Q}(t, y)$ and $\tilde{Q}^{*}(t, y)$ are closed, convex, and satisfy property $(Q)$ in $A$, that the remaining hypotheses of Corollary 8.1 hold and that the sets $\{x\}_{\Omega} \subset S$ and $\left\{u^{\prime \prime}\right\}_{\Omega} \subset T^{\prime \prime}$ are weakly sequentially compact. We do not exclude here that either $U^{\prime}=E_{\alpha}$, or $U^{\prime \prime}=E_{m-\alpha}$, or both.

Remark 8.1. The remarks at the end of the existence theorem of Section 7 hold also for the present extensions. In particular, the present existence theorem can be repeated for generalized solutions $\left(x, p, w, u^{\prime \prime}\right)$ analogous to those introduced in Section 7 with $u^{\prime}$ replaced by $p, w, p=p(t)=$ $\left(p_{1}, \ldots, p_{\mu}\right), w=w(t)=\left(u^{(1)}, \ldots, u^{(\mu)}\right), t \in G$. Then, for

$$
\mu \geqslant \max (r+2, m-\alpha+2)
$$

certainly the corresponding sets $\widetilde{Q}^{\prime}(t, y) \subset E_{r+1}, \widetilde{Q}^{*}(t, y) \subset E_{m-\alpha+1}$ are replaced by the sets $R^{\prime}(t, y)=\operatorname{co} Q^{\prime}(t, y) \subset E_{r+1}, R^{*}(t, y)=\operatorname{co} Q^{*}(t, y) \subset E_{m-x+1}$, 
which are necessarily convex. The present existence theorem then holds with the convexity conditions necessarily satisfied.

\section{Particular Cases}

9.1. Let us assume that $G$ has a smooth boundary $\Gamma=\partial G$ in the sense of Sobolev (or in the sense of Morrey), and take $S=\prod_{i=1}^{n} W_{p_{i}}^{1}(G)$, so that every element $x \in S$ is an $n$-vector function $x(t)=\left(x^{1}, \ldots, x^{n}\right), t \in G$, whose components $x^{i} \in L_{x_{i}}(G), \quad p_{i} \geqslant 1$, possess first-order generalized partial derivatives $\nabla x=\left[\partial x^{i} / \partial t^{j}, i=1, \ldots, n, j=1, \ldots, \nu\right]$ a.e. in $G$, and all $x^{i}$, $\partial x^{i} / \partial t^{j} \in L_{p_{i}}(G), \quad j=1, \ldots, \nu, \quad i=1, \ldots, n$. Let $Y=\prod_{i=1}^{n} L_{p_{i}}(G), \quad V=$ $\prod_{i=1}^{n}\left(L_{p_{i}}(G)\right)^{\nu}$, let $\mathscr{U}: S \rightarrow Y$ be the identity operator, mapping $x \in S$ into $y=x$ as an element of $Y$, and let $\mathscr{L}: S \rightarrow V$ be defined by $\mathscr{L} x=\nabla x$, and thus $s=n, r=n v$. Now, property $(H)$ is trivial. We are now in a position to study the problem of the minimum of a multiple integral

$$
I[x, u]=\int_{G} f_{o}(t, x(t), u(t)) d t,
$$

with side conditions expressed by a total differential (or Dieudonné-Rashevsky) system

$$
\partial x^{i} / \partial t^{j}=f_{i j}(t, x(t), u(t)) \quad \text { a.e. in } G, \quad j=1, \ldots, v, \quad i=1, \ldots, n,
$$

and possible constraints of the form

$$
(t, x(t)) \in A, \quad u(t) \in U(t, x(t)), \quad t \in G .
$$

If $f$ denotes the $n \nu$-vector function $f=\left(f_{i j}\right)$, then the differential system can be written in the form

$$
d x / d t=f(t, x(t), u(t)) .
$$

In this situation, closed classes $\Omega$ such that $\{x\}_{\Omega}$ is weakly sequentially compact can be obtained in a variety of ways, as we have pointed out in Refs. 1-4. For instance, for all $p_{i}>1$, we may define $\Omega$ by means of requirements of the following form: $(\alpha)\left\|x^{i}\right\|_{p_{i}} \leqslant L_{i}$ for given constants $L_{i} ;(\beta)\left\|\partial x^{i} / \partial t^{j}\right\|_{p_{i}} \leqslant L_{i j}$ for given constants $L_{i j} ;(\gamma) I[x, u] \leqslant L_{o}$ for a given constant $L_{o} ;$ and $(\delta)$ boundary conditions concerning the values of the functions $x^{i}$ on suitable parts $\Gamma_{s}$ of the boundary $\Gamma=\partial G$ of $G$. Besides, the sets $U(t, y)$ may be compact and uniformly bounded. 
For all $p_{i}=1$, classes $\Omega$ may be defined by means of analogous requirements; but, if the sets $U(t, y)$ are only closed, then a growth condition may be needed. For instance, in Refs. 3-4 we proposed the following rather general growth condition: $(\epsilon)$ for any $\epsilon>0$, there is an $L$-integrable function $\psi_{\epsilon}(t) \geqslant 0$, $t \in G$, which may depend on $\epsilon$, such that $|f(t, y, u)| \leqslant \psi_{\epsilon}(t)+\epsilon f_{o}(t, y, u)$ for all $(t, y, u) \in M$. Under this hypothesis, for the admissible pairs $x, u$ satisfying a relation $(\gamma)$, the partial derivatives $\partial x^{i} / \partial t^{j}$ are equiabsolutely integrable in $G$. Then, conditions $(\beta)$ are necessarily satisfied with $p_{i}=1$, and any condition $(\alpha)$ makes the class $\{x\}_{\Omega}$ weakly sequentially compact. Besides, under the same hypotheses, boundary conditions as $(\delta)$ are preserved by weak convergence, and the corresponding classes $\Omega$ are closed.

The particular case considered above can be interpreted in the lines of Section 3, by taking $X=\prod_{i=1}^{n} W_{p_{i}}^{1}(G)$, by taking for $X_{o}$ the linear subspace of all $x(t)=\left(x^{1}, \ldots, x^{n}\right), t \in G$, where each $x^{1}$ coincides in $G$ with a function of class $C^{\infty}$ in $E_{\nu}$, and by considering the operators $\mathscr{U}$ and $\mathscr{L}$ defined by $\mathscr{U} x=x$, $\mathscr{L} x=\nabla x$. Let $S$ be the completion of $X_{o}$ by means of the norm $\|x\|=$ $\sqrt{ }\left(\|x\|^{2}+\|\mathscr{U} x\|^{2}+\|\mathscr{L} x\|^{2}\right)$, where $\|x\|,\|\mathscr{U} x\|,\|\mathscr{L} x\|_{\text {are }}$ the norms in $X$, $Y, V$, respectively. It is easy to see that $S$ coincides with $X$, that $\|x\|$ and $\|x\|$ are equivalent norms, and that property $\left(H_{o}\right)$ holds.

9.2. Let us assume that $G$ has a smooth boundary $\Gamma=\partial G$ in the sense of Sobolev, or in the sense of Morrey, of some order $l \geqslant 1$; and this, as we know, does not exclude corner points for $G$. Take $S=\prod_{i=1}^{n} W_{p_{i}}^{l_{i}}(G)$, for some $p_{i} \geqslant 1$, and integer $l_{i}, 1 \leqslant l_{i} \leqslant l, i=1, \ldots, n$. Thus, $x \in S$ is a vector function $x(t)=\left(x^{1}, \ldots, x^{n}\right), t \in G$, with components $x^{i} \in L_{p_{i}}(G)$, and each $x^{i}$ has generalized partial derivatives $D^{\alpha} x^{i}$ of all orders $\alpha, 0 \leqslant|\alpha| \leqslant l_{i}, \alpha=\left(\alpha_{1}, \ldots, \alpha_{y}\right)$, $|\alpha|=\alpha_{1}+\cdots+\alpha_{\nu}, D^{\alpha} x^{i} \in L_{p_{i}}(G)$. Let $s_{i}$ denote the number of multiindices $\alpha$ with $0 \leqslant|\alpha| \leqslant l_{i}-1$, let $s=s_{1}+\cdots+s_{n}$, and let $\mathscr{U}$ denote the operator $\mathscr{U} x=\nabla^{\prime} x=\left\{D^{\alpha} x^{i}, \quad 0 \leqslant|\alpha| \leqslant l_{i}-1, i=1, \ldots, n\right\}$ for $x \in S$, so that $\mathscr{U}: S \rightarrow Y$, where $Y=\prod_{i=1}^{n}\left[L_{p_{i}}(G)\right]^{s_{i}}$. For every $i=1, \ldots, n$, let $\{\alpha\}_{i}$ denote a given collection of distinct multiindices $\alpha$ with $|\alpha|=l_{i}$, let $r_{i} \geqslant 0$ be the number of elements in the collection $\{\alpha\}_{i}$, and let $r=r_{\mathbf{1}}+\cdots+r_{n}$. We take for $\mathscr{L}$ the operator $\mathscr{L} x=\left\{D^{\alpha} x^{i}, \alpha \in\{\alpha\}_{i}, i=1, \ldots, n\right\}$ for $x \in S$, so that $\mathscr{L}: S \rightarrow V$, where $V=\prod_{i=1}^{n}\left[L_{p_{i}}(G)\right]^{r_{i}}$. Thus, $\mathscr{U} x$ is an $s$-vector $y=y(t)=$ $\left(y^{1}, \ldots, y^{s}\right), t \in G$, and $\mathscr{L} x$ is an $r$-vector $v=v(t)=\left(v^{1}, \ldots, v^{r}\right), t \in G$. Again, as in Section 9.1, property $(H)$ is trivial. Let $\nabla^{\prime \prime} x=\left\{D^{\alpha} x^{i},|\alpha|=l_{i}\right.$, $i=1, \ldots, n\}$. We are now in a position to study the problem of the minimum of a multiple integral

$$
I[x, u]=\int_{G} f_{o}\left(t,\left(\nabla^{\prime} x\right)(t), u(t)\right) d t
$$


with side conditions expressed by the system of $r$ partial differential equations

$$
D^{\alpha} x^{i}=f_{i \alpha}\left(t,\left(\nabla^{\prime} x\right)(t), u(t)\right) d t, \quad \alpha \in\{\alpha\}_{i}, \quad i=1, \ldots, n,
$$

and possible constraints of the form

$$
\left(t, \nabla^{\prime} x(t)\right) \in A, \quad u(t) \in U\left(t,\left(\nabla^{\prime} x\right)(t)\right), \quad t \in G .
$$

If $f$ denotes the $r$-vector function $f=\left(f_{i \alpha}, \alpha \in\{\alpha\}_{i}, i=1, \ldots, n\right)$, then the differential system can be written in the form

$$
D x=f\left(t,\left(\nabla^{\prime} x\right)(t), u(t)\right), \quad t \in G,
$$

where $D x=\left(D^{\alpha} x^{i}, \alpha \in\{\alpha\}_{i}, i=1, \ldots, n\right)$.

In this situation, closed classes $\Omega$ such that $\{x\}_{\Omega}$ is weakly sequentially compact can be obtained as in Section 9.1 and we have indicated in Refs. 1-3. For instance, for all $p_{i}>1$, we may define $\Omega$ by means of requirements of the form: $(\alpha)\left\|D^{\alpha} \mathcal{X}^{i}\right\|_{p_{i}} \leqslant L_{i \alpha}, 0 \leqslant|\alpha|=l_{i}-1$, for given constants $L_{i \alpha} ;(\beta)$ $\left\|D^{\alpha} x^{i}\right\|_{p} \leqslant L_{i \alpha},|\alpha|=l_{i}$, for given constants $L_{i \alpha} ;(\gamma) I[x, u] \leqslant L_{o}$ for a given constant $L_{o}$; and $(\delta)$ boundary conditions concerning the values of the functions $x^{i}$ and of the derivatives $D^{\alpha} x^{i}, 0 \leqslant \alpha \leqslant l_{i}-1$, on suitable parts $\Gamma_{s}$ of the boundary $\Gamma=\partial G$ of $G$.

For all $p_{i}=1$, classes $\Omega$ may be defined by means of analogous requirements, and, if the sets $U(t, y)$ are only closed; in conjunction with a growth condition as $(\epsilon)$ in Section 9.1. Under this hypothesis, for the admissible pairs $x$, $u$ satisfying a relation $(\gamma)$, the partial derivatives $D^{\alpha} x^{i}, \alpha \in\{\alpha\}_{i}, i=1, \ldots, n$, are equiabsolutely integrable in $G$, and hence corresponding relations $(\beta)$, for $\alpha \in\{\alpha\}_{i}$, are certainly satisfied for suitable constants $L_{i \alpha}$. For details, see Refs. 1-3.

The present considerations hold even if the collections $\{\alpha\}_{i}$ are made up of arbitrary multiindices $\alpha$ with $0 \leqslant|\alpha| \leqslant l_{i}$.

The situation considered above can also be interpreted in the sense of Section 3, by taking $X=\prod_{i=1}^{n} W_{p_{i}}^{l_{i}}(G)$, by taking for $X_{o}$ the linear subspace of all $x(t)=\left(x^{1}, \ldots, x^{n}\right), t \in G$, where each $x^{i}$ coincides in $G$ with a function of class $C^{\infty}$ in $E_{\nu}$, and by considering the operators $\mathscr{U}, \mathscr{L}$ defined by $\mathscr{U} x=\nabla^{\prime} x$, and $\mathscr{L}=\nabla^{\prime \prime}$. Let $S$ be the completion of $X_{o}$ by means of the norm $\|x\|=$ $\sqrt{ }\left(\|x\|^{2}+\|\mathscr{U} x\|^{2}+\|\mathscr{L} x\|^{2}\right)$, where $\|x\|,\|\mathscr{U} x\|,\|\mathscr{L} x\|$ are the norms in $X$, $Y, V$, respectively. As in Section 9.1, $S$ coincides with $X,\|x\|$ and $\|x \mid\|$ are equivalent norms, and property $\left(H_{o}\right)$ holds.

9.3. Let $G$ be as in Section 9.2, let $X=\prod_{i=1}^{n} W_{p_{i}}^{l_{i}}(G)$ as in Section 9.2, let $X_{o}$ be the linear subspace of all $x(t)=\left(x^{1}, \ldots, x^{n}\right), t \in G$, where each $x^{i}$ coincides 
in $G$ with a function of class $C^{\infty}$ in $E_{v}$, and let $\mathscr{U}: X_{0} \rightarrow Y$ be the operator defined as in Section 9.2 by $\mathscr{U} x=\nabla^{\prime} x=\left[D^{\alpha} x^{i}, 0 \leqslant|\alpha| \leqslant l_{i}-1, i=1, \ldots, n\right]$; hence, $\mathscr{U} x$ is a function $y(t)=\left(y^{1}, \ldots, y^{s}\right) \in Y, t \in G, Y=\prod_{i=1}^{n}\left[L_{p_{i}}(G)\right]^{s_{2}}$, $s=s_{1}+\cdots+s_{n}$. Let $r_{i} \geqslant 0, i=1, \ldots, n$, be arbitrary integers, let $r=r_{1}+\cdots+r_{n}$, let $V=\prod_{i=1}^{n}\left[L_{p_{i}}(G)\right]^{r_{i}}$, and let $\mathscr{L}: X_{o} \rightarrow V$ be any linear differential operator with integrable bounded coefficients in $G$, say $(\mathscr{L} x)^{i}=$ $\sum_{j=1}^{k_{i}} \sum_{s=1}^{n} \sum_{|\alpha|=j} A_{i s j \alpha}(t) D^{\alpha} x^{s}$, of arbitrary orders $k_{i}$ which can be larger than $l_{i}$. Let $S$ be the completion of $X_{o}$ by means of the norm

$$
\|x\|=\sqrt{ }\left(\|x\|^{2}+\|\mathscr{U} x\|^{2}+\|\mathscr{L} x\|^{2}\right),
$$

where $\|x\|,\|\mathscr{U} x\|,\|\mathscr{L} x\|$ are the norms in $X, Y, V$, respectively. If $k_{i}>l_{i}$ for at least one $i$, then certainly $S$ may be distinct from $X$; but, in any case, property $\left(H_{o}\right)$ obviously holds. We are now in a position to consider the problem of the minimum of the multiple integral

$$
I[x, u]=\int_{G} f_{o}\left(t,\left(\nabla^{\prime} x\right)(t), u(t)\right) d t,
$$

with side conditions expressed by the differential system

$$
(\mathscr{L} x)(t)=f\left(t,\left(\nabla^{\prime} x\right)(t), u(t)\right), \quad t \in G, \quad \text { a.e. }
$$

with $f=\left(f_{1}, \ldots, f_{r}\right)$, and possible constraints of the form

$$
\left.\left(t,\left(\nabla^{\prime} x\right)(t)\right) \in A, \quad u(t) \in U\left(t, \nabla^{\prime} x\right)(t)\right), \quad t \in G, \quad \text { a.e. }
$$

Classes $\Omega$ can be defined now as in Section 9.2.

For instance, if we take $\nu=2, n=1, l_{i}=1, m=1, \xi \eta$ coordinates in $E_{2}$, and we take for $\mathscr{L}$ the Laplacian, we may consider the problem of the minimum of the double integral

$$
I[x, u]=\iint_{G} f_{0}(\xi, \eta, x(\xi, \eta), u(\xi, \eta)) d \xi d \eta
$$

with partial differential equation

$$
x_{\xi \xi}+x_{\eta \eta}=f(\xi, \eta, x(\xi, \eta), u(\xi, \eta)), \quad(\xi, \eta) \in G,
$$

and constraints of the forms

$$
(\xi, \eta, x(\xi, \eta)) \in A, \quad u(\xi, \eta) \in U(\xi, \eta, x(\xi, \eta)) .
$$

Here, $X_{o}$ is the linear space of all functions $x(\xi, \eta),(\xi, \eta) \in G$, which coincide 
with some function of class $C^{\infty}$ in $G$, and $S$ is the completion of $X_{o}$ with respect to the norm

$$
\|x\|=\sqrt{ }\left(\|x\|_{p}^{2}+\left\|x_{\xi}\right\|_{p}^{2}+\left\|x_{n}\right\|_{p}^{2}+\left\|x_{\xi \xi}+x_{n \eta}\right\|_{p}^{2}\right) .
$$

The minimum above is sought in classes $\Omega$ of elements $x \in S$.

9.4. Let us take in the existence theorem $r=m, U(t, y)=E_{r}$, $A=\operatorname{cl} G \times E_{s}, M=A \times E_{r}, f(t, y, u)=u$, that is, $f_{i}=u^{i}, i=1, \ldots, r$. Then, the abstract Lagrange problem of the existence theorem reduces to the free problem of the minimum of the multiple integral

$$
I[x]=\int_{G} f_{0}(t,(\mathscr{U} x)(t),(\mathscr{L} x)(t)) d t,
$$

which was considered by Fichera (Refs. 8-10). Note that here $\mathscr{L} x=u$, that is, the element $u \in T$ is uniquely determined by the element $x \in S$ (in an admissible pair $x, u)$. Here, the sets $\mathscr{Q}(t, y)$ are the sets

$$
\tilde{Q}(t, y)=\left[\left(z^{o}, u\right) \mid z^{o} \geqslant f_{o}(t, y, u), u \in E_{r}\right] \subset E_{r+1},
$$

that is, the sets of all points on or above the figurative $f_{o}$. Thus, the sets $\widetilde{Q}(t, y)$, are convex if, and only if, $f_{o}(t, y, u)$ is convex in $u$ for every $(t, y) \in A$. The following growth condition has been consistently used by Tonelli, Morrey, Fichera, and Cesari: $(\Phi)$ there is a continuous scalar function $\Phi(z)$, $0 \leqslant z<+\infty$, such that $\Phi(z) / z \rightarrow+\infty$ as $z \rightarrow+\infty$, and $f_{0}(t, y, u) \geqslant \Phi(|u|)$ for all $(t, y, u) \in M$. Under growth condition $(\Phi)$, the set $\bar{Q}(t, y)$, if convex, certainly are closed and satisfy property $(Q)$ in $A$ (see Refs. 14-15). Theorems 6.1 and 7.1 and Corollary 6.1 essentially contain the corresponding lower semicontinuity theorem and the existence theorem of Fichera (Refs. 8-10).

9.5. If we take $r=m=\nu n, s=n, U(t, y)=E_{r}, A=\operatorname{cl} G \times E_{n}$, $M=A \times E_{v n}, f(t, y, u)=u, S=\prod_{i=1}^{n} W_{p_{i}}^{1}(G), Y, V$ as in Section 9.1, $\mathscr{U}: X \rightarrow Y$, the identity, $\mathscr{L}=\nabla$ as in Section 9.1 , then we have the free problem of the minimum of the multiple integral

$$
I[x]=\int_{G} f_{o}(t, x(t),(\nabla x)(t)) d t,
$$

considered by Morrey (Ref. 11). Again, as in Section 9.4, $\nabla x=u$, that is, the element $u \in T$ is uniquely determined by the element $x \in S$ (in an admissible pair $x, u)$. As in Section 9.4, the sets $\widetilde{Q}(t, y)$ are the sets of all points on or above the figurative $f_{o}$, and hence they are convex if, and only if, the function $f_{o}(t, y, u)$ is convex in $u$ for every $(t, y) \in A$. Again, the growth condition $(\Phi)$ 
guarantees that the sets $\tilde{Q}(t, y)$, if convex, are also closed and satisfy property $(Q)$ in $A$. Here, as in Section 9.4, Theorems 6.1 and 8.1 and Corollary 6.1 essentially contain the corresponding lower semicontinuity theorem and existence theorem of Morrey (Ref. 11).

9.6. Let us assume that $G$ has a smooth boundary $\Gamma=\partial G$ (as in Section 9.1 above), take $S=\left[W_{p}{ }^{1}(G)\right]^{n}$ for a given $p>2$, so that every element $x \in S$ is an $n$-vector function $x(t)=\left(x^{1}, \ldots, x^{n}\right), t \in G \subset E_{\nu}$, whose components $x^{i} \in L_{p}(G), p>2$, possess first-order generalized partial derivatives $\nabla x=\left[\partial x^{i} / \partial t^{s}, i=1, \ldots, n, j=1, \ldots, \nu\right]$ a.e. in $G$, and all $x^{i}, \partial x^{i} / \partial t^{s} \in L_{p}(G)$. Let $Y=\left[L_{p}(G)\right]^{n}, V=L_{p / 2}(G)$, let $s=n, r=1$, let $\mathscr{U}: S \rightarrow Y$ be the identity operator mapping $x \in S$ into $y=x$ as an element of $Y$, and let $\mathscr{L}: S \rightarrow V$ be the nonlinear differential operator defined by $\mathscr{L} x=$ $\sum_{i, j=1}^{n} \sum_{s=1}^{v} a_{i j s}(t) x^{i}\left(\partial x^{j} / \partial t^{s}\right)$ for $x \in S$, where $a_{i j s}(t)$ are given measurable bounded functions on $G$. If $x \in S$, then each product $a_{i j s}(t) x^{i}\left(\partial^{i} x / \partial t^{s}\right)$ is in $L_{p / 2}(G)$. Also, if $x, x_{k} \in S, k=1,2, \ldots$, and $x_{k} \rightarrow x$ weakly in $S$, then $x_{k c}{ }^{i} \rightarrow x^{i}$ strongly in $L_{p}(G), \partial x_{k}{ }^{i} / \partial t^{s} \rightarrow \partial x^{i} / \partial t^{s}$ weakly in $L_{p}(G)$ as $k \rightarrow \infty, i=1, \ldots, n$, $s=1, \ldots, \nu$, and $\mathscr{L} x_{k} \rightarrow x$ weakly in $L_{p / 2}(G)$. Here, axiom $(H)$ is trivially satisfied, and we take for $T$ the set of all $m$-vector functions $u(t)=\left(u^{1}, \ldots, u^{m}\right)$, $t \in G$, measurable in $G$. Here, $f_{o}(t, y, u)$ and $f(t, y, u)$ are both scalar functions on $M$. We deal here with the minimum of a functional

$$
I[x, u]=\int_{G} f_{o}(t, x(t), u(t)) d t,
$$

with partial differential equation and constraints

$$
\begin{gathered}
\sum_{i, j=1}^{n} \sum_{s=1}^{v} a_{i j s}(t) x^{i}\left(\partial x^{j} / \partial t^{s}\right)=f(t, x(t), u(t)), \\
(t, x(t)) \in A, \quad u(t) \in U(t, x(t)), \quad \text { a.e. in } G .
\end{gathered}
$$

The sets $\widetilde{Q}$ are now subsets of $E_{2}$,

$$
\widetilde{Q}(t, x)=\left[\left(z^{o}, z\right) \mid z^{0} \geqslant f_{o}(t, x, u), z=f(t, x, u), u \in U(t, x)\right],
$$

and these sets shall be assumed to be closed, convex, and satisfying property $(Q)$ in $A$.

In the present situation, closed classes $\Omega$ such that $\{x\}_{\Omega}$ is weakly sequentially compact can be obtained by requirements of the form $(\alpha)\left\|x^{i}\right\|_{p} \leqslant L_{i}$ for given constants $L_{i},(\beta)\left\|\partial x^{i} / \partial t^{j}\right\|_{p} \leqslant L_{i j}$ for given constants $L_{i j}$, and other requirements as $(\gamma)$ and $(\delta)$ in Section 9.1 above. 
9.7. Let $p>2, G, Y, V$ be as in Section 9.6 above, let $S=\left[W_{p}{ }^{2}(G)\right]^{n}$, let $u=\left(u^{\prime}, u^{\prime \prime}\right)$ with $u^{\prime}=\left(u^{1}, \ldots, u^{\alpha}\right), u^{\prime \prime}=\left(u^{\alpha+1}, \ldots, u^{m}\right)$, and $u \in T=T^{\prime} \times T^{\prime \prime}$, where $T^{\prime}$ is the set of all $\alpha$-vector functions $u^{\prime}(t), t \in G$, which are measurable in $G$, and $T^{\prime \prime}=\left[L_{p}(G)\right]^{m-\alpha}$ with norm $\left\|u^{\prime \prime}\right\|=\sqrt{ }\left(\sum_{j=\alpha+1}^{m}\left\|u^{j}\right\|_{L_{p}}^{2}\right)$. Let $\mathscr{U}: S \rightarrow Y$ be the identity operator as in Section 9.6, and let $\mathscr{L}: S \times T^{\prime \prime} \rightarrow V$ be the operator defined by

$$
\mathscr{L}\left(x, u^{\prime \prime}\right)=\Delta x+\sum_{i=\alpha+1}^{m} \sum_{j=1}^{n} \sum_{s=1}^{\nu} a_{i j s}(t) u^{i}(t)\left(\partial x^{j} / \partial t^{s}\right),
$$

where $x \in S, u^{\prime \prime} \in T$, and all $a_{i j s}(t)$ are bounded measurable functions on $G$, and where $\Delta x$ denotes the sum of the second-order partial derivatives $\partial^{2} x^{i} /\left(\partial t^{s}\right)^{2}, i=1, \ldots, n, s=1, \ldots, \nu$. Then, as in Section 9.6, if $x \in S, u \in T^{\prime \prime}$, then $\mathscr{L}\left(x, u^{\prime \prime}\right) \in L_{p / 2}(G)$; and, if $x_{k} \rightarrow x$ weakly in $S$ and $u_{k}^{\prime \prime} \rightarrow u^{\prime \prime}$ weakly in $T$, then $\mathscr{L}\left(x_{k}, u_{k}^{\prime \prime}\right) \rightarrow \mathscr{L}\left(x, u^{\prime \prime}\right)$ weakly in $L_{p / 2}(G)$. Axiom $\left(H^{*}\right)$ of Section 8 is trivially satisfied. We deal here with the minimum of a functional

$$
I\left[x, u^{\prime}, u^{\prime \prime}\right]=\int_{G} f_{o}\left(t, x(t), u^{\prime}(t)\right) d t,
$$

with partial differential equation and constraints

$$
\begin{aligned}
& \Delta x+\sum_{i=\alpha+1}^{m} \sum_{j=1}^{n} \sum_{s=1}^{D} a_{i j s}(t) u^{i}(t)\left(\partial x^{j} / \partial t^{s}\right)=f\left(t, x(t), u^{\prime}(t)\right), \\
& (t, x(t)) \in A, \quad u^{\prime}(t) \in U^{\prime}(t, x(t)), \quad u^{\prime \prime}(t) \in U^{\prime \prime}(t, x(t)),
\end{aligned}
$$

a.e. in $G$, and, as mentioned, $x \in S, u^{\prime} \in T^{\prime}, u^{\prime \prime} \in T^{\prime \prime}$. We shall now consider the sets $\widetilde{Q}^{\prime}(t, x)=\left[\left(z^{o}, z\right) \mid z^{o} \geqslant f_{o}\left(t, x, u^{\prime}\right), z=f\left(t, x, u^{\prime}\right), u^{\prime} \in U^{\prime}(t, x)\right]$, and these sets as well as the sets $U^{\prime \prime}(t, x)$, shall be assumed to be closed, convex, and satisfying property $(Q)$ in $A$. We do not exclude here that either $U^{\prime}=E_{\alpha}$, or $U^{\prime \prime}=E_{m-\alpha}$, or both. Note that, in the notations of Section 8 , we have here $f^{\prime \prime}=0, f_{o}^{\prime \prime}=0$.

In the present situation, closed classes $\Omega$ such that $\{x\}_{\Omega}$ and $\left\{u^{\prime \prime}\right\}_{\Omega}$ are weakly sequentially compact, as requested in Section 8 , can be obtained by requirements of the forms $(\alpha)\left\|x^{j}\right\|_{p} \leqslant L_{j}, j=1, \ldots, n,(\beta)\left\|\partial x^{j} \mid \partial t^{s}\right\|_{p} \leqslant L_{j s}$, $\left\|\partial^{2} x^{j} / \partial t^{s} \partial t^{\sigma}\right\|_{p} \leqslant L_{j s \sigma}, \quad j=1, \ldots, n, \quad s, \quad \sigma=1, \ldots, \nu,\left(\beta^{\prime}\right)\left\|u^{i}\right\|_{p} \leqslant L_{i}, \quad i=$ $\alpha+1, \ldots, m$, for given constants $L_{j}, L_{j s}, L_{i}$, and by requirements as $(\gamma)$ and $(\delta)$ in Sections 9.1 and 9.6 above.

9.8. Let $v=2, n=1, m=1, \alpha=0, p=2, G=[0 \leqslant \xi, \eta \leqslant 1]$, $Y=L_{2}(G), \quad V=L_{1}(G), \quad V_{o}=L_{1}(G), \quad r=1, s=1, \quad S=W_{2}^{1}(G), \quad U=$ 
$U^{\prime \prime}=E_{1}, T=T^{\prime \prime}=L_{2}(G)$. We consider here the problem of the minimum of the double integral

$$
I[x, u]=\iint_{G}\left[E(\xi, \eta) x^{2}+F(\xi, \eta) u\right] d \xi d \eta,
$$

in the class $\Omega$ of all pairs $x, u$, with $x \in S, u \in T$, satisfying the first-order partial differential equation

$$
A(\xi, \eta) x_{\xi}+B(\xi, \eta) x_{\eta}+C(\xi, \eta) x u+D(\xi, \eta) u=0 \quad \text { a.e. in } G
$$

the boundary condition $x(0, \eta)=1,0 \leqslant \eta \leqslant 1$, and the constraints $\|u\|_{L_{2}} \leqslant 1$, $\|x\|_{L_{2}} \leqslant 1,\left\|x_{\xi}\right\|_{L_{2}} \leqslant 1,\left\|x_{\eta}\right\|_{L_{2}} \leqslant 1$. The class $\Omega$ is nonempty since $x=1$, $u=0$ is in $\Omega$. Here, $A, B, C, D, E, F$ denote given measurable essentially bounded real-valued functions in $G$. Note that the sets $\{x\}_{\Omega},\{u\}_{\Omega}$ are weakly compact in the respective spaces $S=W_{2}^{1}(G), T=T^{\prime \prime}=L_{2}(G)$. Here, we take $\mathscr{U} x=x, \quad \mathscr{L}(x, u)=A x_{\xi}+B x_{\eta}+C x u+D u, \quad \mathscr{L}_{0}(x, u)=E x^{2}+F u$; hence, $\mathscr{U}: S \rightarrow Y, \mathscr{L}: S \times T \rightarrow V, \mathscr{L}_{0}: S \times T \rightarrow V_{0}$. If $x_{k} \rightarrow x$ weakly in $S=W_{2}{ }^{1}$ and $u_{k} \rightarrow u$ weakly in $T=T^{\prime \prime}=L_{2}$, then $x_{k} \rightarrow x$ strongly in $L_{2}$, $x_{k}{ }^{2} \rightarrow x$ strongly in $L_{1}, x_{k} u_{k} \rightarrow x u$ weakly in $L_{1}, \mathscr{U} x_{k} \rightarrow \mathscr{U} x$ strongly in $Y=L_{2}, \mathscr{L}\left(x_{k}, u_{k}\right) \rightarrow \mathscr{L}(x, u)$ weakly in $V=L_{1}$, and $\mathscr{L}_{0}\left(x_{k}, u_{k}\right) \rightarrow \mathscr{L}_{0}(x, u)$ weakly in $V_{o}=L_{1}$. Here we take $f_{o}=0$, the requirements concerning the sets $\tilde{Q}$ are vacuous, and we take $U^{\prime \prime}=E_{1}$, a fixed convex, closed set. Also, we can take $\psi=0$. In view of Section 8 , the integral $I$ above has an absolute minimum in $\Omega$.

9.9. Let $\nu=2, n=1, m=1, \alpha=0, p=2, G=[0 \leqslant \xi, \eta \leqslant 1]$, $Y=L_{2}(G)^{3}, \quad V=L_{1}(G), \quad V_{o}=L_{1}(G), r=1, s=3, S=W_{2}{ }^{2}(G), U=$ $U^{\prime \prime}=E_{1}, T=T^{\prime \prime}=L_{2}(G)$. We consider here the problem of the minimum of the double integral

$$
I[x, u]=\iint_{G}\left[F(\xi, \eta) x_{\xi}^{2}+G(\xi, \eta) x_{\eta}{ }^{2}+H(\xi, \eta) u\right] d \xi d \eta
$$

in the class $\Omega$ of all pairs $x, u$, with $x \in S, u \in T$, satisfying the second-order partial differential equation

$$
A(\xi, \eta) x_{\xi \xi}+B(\xi, \eta) x_{\eta \eta}+\left[C(\xi, \eta) x_{\xi}+D(\xi, \eta) x_{\eta}+E(\xi, \eta)\right] u=0
$$

a.e. in $G$, boundary conditions $x=1$ on the boundary $\partial G$ of $G$, and the constraints $\|u\|_{L_{2}} \leqslant 1,\|x\|_{W_{2}}{ }^{2} \leqslant 1$. The class $\Omega$ is nonempty since $x=1$, 
$u=0$ is in $\Omega$. Here $A, B, C, D, E, F, G, H$ denote given measurable essentially bounded real-valued functions in $G$. Note that the sets $\{x\}_{\Omega},\{u\}_{\Omega}$ are weakly compact in the respective space $S=W_{2}{ }^{2}(G), T=T^{\prime \prime}=L_{2}(G)$. Here we take $\mathscr{U} x=\left[x, x_{\xi}, x_{\eta}\right], \mathscr{L}(x, u)=A x_{\xi \xi}+B x_{\eta \eta}+\left(C x_{\xi}+D x_{\eta}+E\right) u, \mathscr{L}_{0}(x, u)=$ $F x_{\xi}{ }^{2}+G x_{\eta}{ }^{2}+H u$; hence, $\mathscr{U}: S \rightarrow Y, \mathscr{L}: S \times T \rightarrow V, \mathscr{L}_{0}: S \times T \rightarrow V_{o}$. If $x_{k} \rightarrow x$ weakly in $S=W_{2}^{2}$ and $u_{k} \rightarrow u$ weakly in $T=T^{\prime \prime}=L_{2}$, then $\left(x_{k}, x_{k \xi}, x_{k \eta}\right) \rightarrow\left(x, x_{\xi}, x_{\eta}\right)$ strongly in $\left(L_{2}\right)^{3}, x_{k \xi}^{2} \rightarrow x_{\xi}{ }^{2}, x_{k \eta}^{2} \rightarrow x_{\eta}{ }^{2}$ strongly in $L_{1}, x_{k \xi} u_{k} \rightarrow x_{\xi} u, x_{k \eta} u_{k} \rightarrow x_{\eta} u$ weakly in $L_{1}$. Finally, $\mathscr{U} x_{k} \rightarrow \mathscr{U} x$ strongly in $Y$, $\mathscr{L}\left(x_{k}, u_{k}\right) \rightarrow \mathscr{L}(x, u)$ weakly in $V, \mathscr{L}_{o}\left(x_{k}, u_{k}\right) \rightarrow \mathscr{L}_{o}(x, u)$ weakly in $V_{o}$. Here, we take $f_{o}=0$, the requirement concerning the sets $\mathscr{Q}$ are vacuous, and we take $U^{\prime \prime}=E_{1}$, a fixed convex closed set. Also, we take $\psi=0$. In view of Section 8 , the integral $I$ above has an absolute minimum in $\Omega$.

9.10. Let $G$ be any bounded open subset of the $t$-space $E_{\nu}, t=\left(t^{1}, \ldots, t^{v}\right)$, let $S=\left[L_{p}(G)\right]^{n}$ for given $p>1$ and $n \geqslant 1$, let $K(t, s)$ be a given $s \times n$ kernel matrix defined and continuous in $\mathrm{cl} G \times \mathrm{cl} G$, and let $\mathscr{U}, \mathscr{L}$ be defined by

$$
(\mathscr{U} x)(t)=\int_{G} K(t, s) x(s) d s, \quad \mathscr{L}_{x}=x,
$$

hence $\mathscr{U}: S \rightarrow Y=\left[L_{p}(G)\right]^{s}, \mathscr{L}: S \rightarrow V=S=\left[L_{p}(G)\right]^{n}$. If $x_{k} \rightarrow x$ weakly in $S$, then $\mathscr{U} x_{k} \rightarrow \mathscr{U} x$ strongly in $L_{p}$, and $\mathscr{L} x_{k} \rightarrow \mathscr{L} x$ weakly in $V$. We consider here the problem of the minimum of the multiple integral

$$
\left.I[x]=\int_{G} f_{o}(t, \mathscr{U} x)(t), x(t)\right) d t .
$$

We assume here that $f_{o}(t, y, v)$, or $f_{o}: \operatorname{cl} G \times E_{s} \times E_{n} \rightarrow E_{1}$, be a given continuous real-valued function, convex in $v, v=\left(v^{1}, \ldots, v^{n}\right)$, and satisfying a growth condition of the form $f_{o}(t, y, v) \geqslant \Phi(|v|)$. Here, $\Phi(\xi), 0 \leqslant \xi<+\infty$, is a given real-valued continuous function with $\Phi(\xi) / \xi \rightarrow+\infty$ as $\xi \rightarrow+\infty$. Then, for some constant $c \geqslant 0$, we have $\Phi \geqslant-c$ and, hence, $f_{o} \geqslant-\psi(t)$ with $\psi=c$, a constant function. In view of Section 7 , the integral above has an absolute minimum in $S$. This example was considered by Fichera in Ref. 9. In view of the same Section 7 , the integral $I[x]$ has an absolute minimum also in the class $\Omega$ of all elements $x \in S$ satisfying a relation

$$
\int_{G} K_{1}(t, s) x(s) d s=f_{1}(t) \quad \text { a.e. in } G,
$$

where $f_{1}$ is a given $r$-vector continuous function in $\mathrm{cl} G$ and $K_{1}(t, s)$ is a given $r \times n$ kernel matrix defined and continuous in $\mathrm{cl} G \times \mathrm{cl} G$. 


\section{References}

1. CESARI, L., Existence Theorems for Multidimensional Problems of Optimal Control, Differential Equations and Dynamical Systems, Edited by J. K. Hale and J. P. LaSalle, Academic Press, New York, 1967.

2. Cesari, L., Existence Theorems for Multidimensional Lagrange Problems, Journal of Optimization Theory and Applications, Vol. 1, No. 2, 1967.

3. Cesari, L., Sobolev Spaces and Multidimensional Lagrange Problems of Optimization, Annali della Scuola Normale Superiore di Pisa, Vol. 22, No. 3, 1968.

4. CESARI, L., Multidimensional Lagrange Problems of Optimization in a Fixed Domain and an Application to a Problem of Magnetohydrodynamics, Archive for Rational Mechanics and Analysis, Vol. 29, No. 2, 1968.

5. Tonelli, L., Sur la Semi-Continuité des Intégrales Doubles du Calcul des Variations, Acta Mathematica, Vol. 53, 1929.

6. Tonelli, L., L'Estremo Assoluto degli Integrali Doppi, Annali della Scuola Normale Superiore di Pisa, Vol. 2, No. 2, 1933.

7. Tonelli, L., Opere Scelte, Vol. 3, Edizioni Cremonese, Roma, Italy, 1962.

8. Fichera, G., Problemi Elastostatici con Vincoli Unilaterali: il Problema di Signorini con Ambigue Condizioni al Contorno, Memorie della Accademia Nazionale dei Lincei, Vol. 7, No. 8, 1964.

9. Fichera, G., Semicontinuity of Multiple Integrals in Ordinary Form, Archive for Rational Mechanics and Analysis, Vol. 17, No. 5, 1964.

10. Fichera, G., Semicontinuitá ed Esistenza del Minimo per una Classe di Integrali Multipli, Revue Roumaine de Mathématiques Pures et Appliquées, Vol. 12, No. 9, 1967.

11. Morrey, C. B., Multiple Integral Problems in the Calculus of Variations and Related Topics, University of California Publications in Mathematics, New Series, 1943.

12. Lions, J. L., Contrôle Optimal de Systèmes Gouvernés par des Équations aux Dérivées Partielles, Dunod, Paris, France, 1968.

13. MCShane, E. J., and Warfield, R. B., On Filippov's Implicit Functions Lemma, Proceedings of the American Mathematical Society, Vol. 18, No. 1, 1967.

14. Cesari, L., Existence Theorems for Weak and Usual Optimal Solutions in Lagrange Problems with Unilateral Constraints. I and II, Transactions of the American Mathematical Society, Vol. 124, No. 3, 1966.

15. CESARI, L., Existence Theorems for Optimal Controls of the Mayer Type, SIAM Journal on Control, Vol. 6, No. 4, 1968.

16. Cesari, L., Closure, Lower Closure, and Semicontinuity Theorems in Problems of Optimal Control, SIAM Journal on Control (to appear). 
17. Cesari, L., Seminormality and Upper Semicontinuity in Optimal Control, Journal of Optimization Theory and Applications, Vol. 6, No. 2, 1970.

18. Olech, O., Existence Theorems for Optimal Problems with Vector-Valued Cost Functions, Transactions of the American Mathematical Society, Vol. 136, 1967.

19. Olech, O., Existence Theorems for Optimal Control Problems Involving Multiple Integrals, Journal of Differential Equations, Vol. 6, No. 3, 1969. 